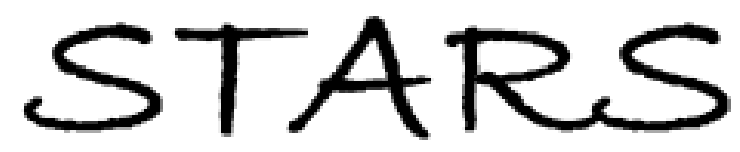

University of Central Florida

STARS

$1-1-2012$

\title{
Breakup and coalescence characteristics of a hollow cone swirling spray
}

\author{
Abhishek Saha \\ University of Central Florida \\ Joshua D. Lee \\ University of Central Florida \\ Saptarshi Basu
}

Find similar works at: https://stars.library.ucf.edu/facultybib2010 University of Central Florida Libraries http://library.ucf.edu

This Article is brought to you for free and open access by the Faculty Bibliography at STARS. It has been accepted for inclusion in Faculty Bibliography 2010 s by an authorized administrator of STARS. For more information, please contactSTARS@ucf.edu.

\section{Recommended Citation}

Saha, Abhishek; Lee, Joshua D.; and Basu, Saptarshi, "Breakup and coalescence characteristics of a hollow cone swirling spray" (2012). Faculty Bibliography 2010s. 3229.

https://stars.library.ucf.edu/facultybib2010/3229

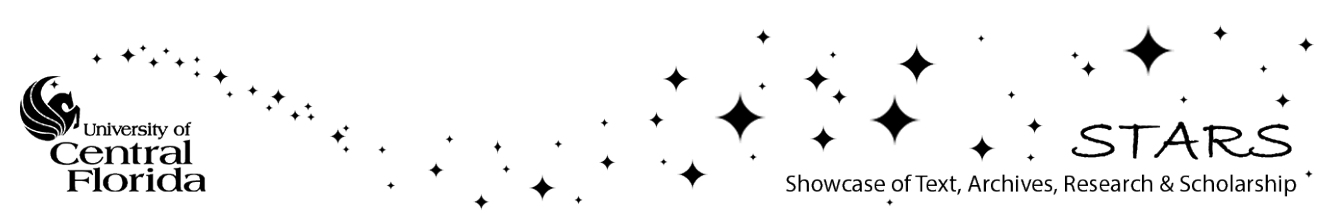




\section{Breakup and coalescence characteristics of a hollow cone swirling spray}

Abhishek Saha, Joshua D. Lee, Saptarshi Basu, and Ranganathan Kumar

Citation: Physics of Fluids 24, 124103 (2012); doi: 10.1063/1.4773065

View online: https://doi.org/10.1063/1.4773065

View Table of Contents: http://aip.scitation.org/toc/phf/24/12

Published by the American Institute of Physics

\section{Articles you may be interested in}

Analyses of Kolmogorov's model of breakup and its application into Lagrangian computation of liquid sprays under air-blast atomization

Physics of Fluids 15, 184 (2003); 10.1063/1.1527914

Large eddy simulation of spray atomization with stochastic modeling of breakup

Physics of Fluids 22, 115106 (2010); 10.1063/1.3508353

Mechanism of atomization of a liquid jet

The Physics of Fluids 25, 1730 (1982); 10.1063/1.863650

Sheet, ligament and droplet formation in swirling primary atomization

AIP Advances 8, 045211 (2018); 10.1063/1.5017162

Quadratic formula for determining the drop size in pressure-atomized sprays with and without swirl Physics of Fluids 28, 063302 (2016); 10.1063/1.4951666

Planar liquid jet: Early deformation and atomization cascades

Physics of Fluids 29, 062109 (2017); 10.1063/1.4986790

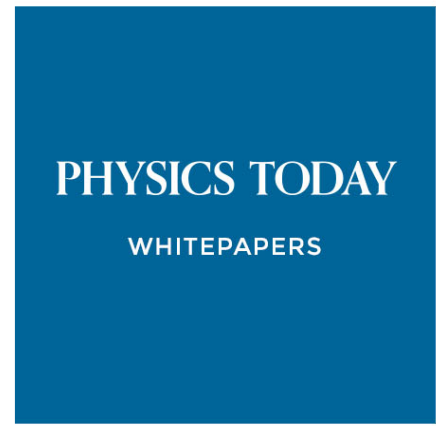

ADVANCED LIGHT CURE ADHESIVES

Take a closer look at what these environmentally friendly adhesive systems can do

\section{READ NOW}

PRESENTED BY Q8 MASTERBOND 


\title{
Breakup and coalescence characteristics of a hollow cone swirling spray
}

\author{
Abhishek Saha, ${ }^{1, a)}$ Joshua D. Lee, ${ }^{1, a)}$ Saptarshi Basu, ${ }^{2, a)}$ \\ and Ranganathan Kumar',a),b) \\ ${ }^{1}$ University of Central Florida, Orlando, Florida 32816, USA \\ ${ }^{2}$ Indian Institute of Science, Bangalore, Karnataka 560012, India
}

(Received 25 June 2012; accepted 3 December 2012; published online 27 December 2012)

\begin{abstract}
This paper deals with an experimental study of the breakup characteristics of water emanating from hollow cone hydraulic injector nozzles induced by pressure-swirling. The experiments were conducted using two nozzles with different orifice diameters $0.3 \mathrm{~mm}$ and $0.5 \mathrm{~mm}$ and injection pressures $(0.3-4 \mathrm{MPa})$ which correspond to $\mathrm{Re}_{\mathrm{p}}$ $=7000-26000$. Two types of laser diagnostic techniques were utilized: shadowgraph and phase Doppler particle anemometry for a complete study of the atomization process. Measurements that were made in the spray in both axial and radial directions indicate that both velocity and average droplet diameter profiles are highly dependent on the nozzle characteristics, Weber number and Reynolds number. The spatial variation of diameter and velocity arises principally due to primary breakup of liquid films and subsequent secondary breakup of large droplets due to aerodynamic shear. Downstream of the nozzle, coalescence of droplets due to collision was also found to be significant. Different types of liquid film breakup were considered and found to match well with the theory. Secondary breakup due to shear was also studied theoretically and compared to the experimental data. Coalescence probability at different axial and radial locations was computed to explain the experimental results. The spray is subdivided into three zones: near the nozzle, a zone consisting of film and ligament regime, where primary breakup and some secondary breakup take place; a second zone where the secondary breakup process continues, but weakens, and the centrifugal dispersion becomes dominant; and a third zone away from the spray where coalescence is dominant. Each regime has been analyzed in detail, characterized by timescale and Weber number and validated using experimental data. (c) 2012 American Institute of Physics. [http://dx.doi.org/10.1063/1.4773065]
\end{abstract}

\section{INTRODUCTION}

In spray nozzles, atomization can be achieved predominantly in two ways, either by discharging liquid at relative high velocities into a slow moving environment (hydraulic nozzles) or by discharging liquid at low velocities into a fast moving gas flow (air-blast nozzles). These two types of injector nozzles are featured in majority of the industry applications such as power generation, food or pharmaceutical powder formation, spray painting, petroleum refining, and thermal sprays. Each nozzle is suitable for a particular application. Selecting the correct nozzle for a given application is critical for optimal design. Most of the aero-engines and IC engines use liquid fuel and different atomization techniques to obtain a homogenous mixture at different equivalence ratios. Depending on the design of the pre-vaporizers and combustors, different nozzles are used in engines. The most common atomizer used in these combustors is the pressure-swirl nozzle (Simplex nozzle). Moreover, among different designs of pressure-swirl nozzles, hollow cone nozzles are preferred in

\footnotetext{
a) A. Saha, J. D. Lee, S. Basu, and R. Kumar contributed equally to this work.

b) Author to whom correspondence should be addressed. Electronic mail: ranganathan.kumar@ucf.edu.
} 
combustion applications due to the comparatively uniform liquid distribution in the radial direction. This uniform liquid distribution helps to enhance and homogenize air-fuel mixture which improves the combustion performance and reduces pollutant emission. Research performed in the last two decades was focused on developing and improving the numerical models which predict the droplet distribution profiles. The experimental studies performed with pressure-swirl nozzles have reported contradictory results over the last few years. ${ }^{1}$ Thus, the fundamentals of spray dynamics, such as spray formation, liquid breakup length, and droplet breakup regimes still need to be understood properly for a pressure-swirl nozzle.

In spray nozzles, liquid comes out in the form of a liquid sheet or film. This film goes through different aerodynamic instabilities to disintegrate into ligaments and eventually droplets. In a pressureswirl nozzle, with increase in pressure, the spray profile changes and it is observed that before the spray becomes fully developed, the liquid must pass through four stages of spray development; dribble stage, distorted pencil stage, onion stage, and Tulip stage ${ }^{2}$ where the last three stages are usually dominated by long wavelength film breakup. Once the spray becomes fully developed, short wavelength film breakup becomes dominant. This was shown analytically $y^{3,4}$ using a linear instability analysis which allowed the prediction of film length based on Weber number which correlated to a certain type of wavelength breakup. Once the Kelvin-Helmholtz instabilities and wave propagation attain the maximum growth, the liquid sheet will shear off to produce liquid ligaments beginning the onset of the breakup process.

The atomization process can be described as the mechanism to increase surface to mass ratio in the liquid phase in order to enhance the vaporization rate and drag force. ${ }^{5}$ The breakup process itself consists of two steps: primary and secondary breakups. The primary atomization process is generally controlled by initial disturbances in the liquid-gas interface and the mechanism that allows these disturbances to grow. Thus, during primary atomization, the liquid film emanating from the nozzle undergoes hydrodynamic instabilities and relatively large drag forces that cause the formation of ligaments or other irregular liquid elements, followed by the formation of droplets. A secondary breakup regime follows, which is caused by the aerodynamic instabilities resulting in larger droplets deforming and breaking up into smaller daughter droplets. ${ }^{1,6,7}$ In order to understand the breakup process for hydraulic nozzles and to establish models for droplet distributions for liquid jet nozzles, researchers have used various optical techniques such as phase Doppler particle anemometry (PDPA) and Laser Doppler Velocimetry (LDV) to investigate the effects of various system parameters, i.e., viscosity, density, and surface tension on the distribution of droplet diameters and velocities..$^{7-10}$

Aliseda et al. ${ }^{11}$ developed a model for a coaxial atomizer generally used in pharmaceutical industry to predict the diameter distribution for non-Newtonian liquids. Butler Ellis et al. ${ }^{12}$ and Bolszo et al. ${ }^{13}$ reported the effect of oil-water emulsion on atomization characteristics for pressure swirl nozzles. They identified conditions at which the emulsion becomes unstable and the droplets contain only a single liquid phase. For Newtonian liquids, it has been reported that system parameters, such as viscosity, density, and nozzle diameter affects the spreading (cone) angle and breakup. ${ }^{14}$ Theoretical analysis on deformation and distortion due to several instabilities has been summarized by Reitz and Bracco, ${ }^{15}$ Lin and Reitz, ${ }^{16}$ Sirignano and Mehring, ${ }^{17}$ Lasheras et al. ${ }^{18}$ Lasheras and Hopfinger, ${ }^{19}$ Senecal et al.,${ }^{3}$ Domouchel and group. ${ }^{20-22}$ Senecal et al. ${ }^{3}$ and Schmidt et al. ${ }^{4}$ reported two distinct regimes of film atomization for cylindrical jet hydraulic nozzles; long and short wave induced film breakup similar to the findings by Sivakumar and Kulkarni ${ }^{23}$ who reported five regimes for air-blast nozzles. Senecal et al. ${ }^{3}$ also found that different types of film breakups are dependent on Weber number. Using high speed imaging, Wahono et al. ${ }^{24}$ qualitatively visualized the spray structure to understand the types of instabilities and ligament formations that are exhibited in hydraulic jet nozzles. No significant model development or validation was done with these experiments that correlated the effects of injection pressure on droplet profiles and breakup and coalescence regimes in a pressure-swirl induced hydraulic nozzle.

The main objectives of this research are to experimentally investigate the effects of Reynolds number and Weber number on the liquid breakup regimes including droplet coalescence in two cylindrical hollow cone pressure-swirl hydraulic atomizing nozzles. This study will identify the spray characteristics and droplet diameter and velocity profiles for each nozzle at different Reynolds number (injection pressure) in the axial and radial direction. This paper uses the theory of liquid 


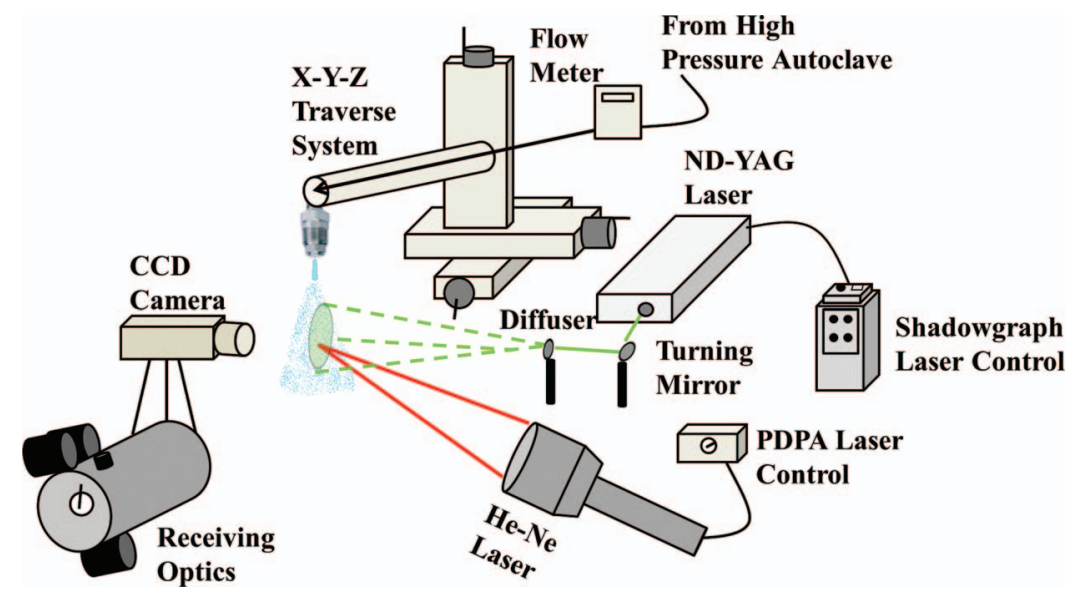

FIG. 1. Experimental setup.

film breakup and shows how the long and short wavelength instabilities are important for low and high Weber number ranges, respectively. The dependence of liquid breakup characteristics has been validated with experimental data for the first time in the context of a pressure-swirl nozzle. Coalescence probability and swirl induced dispersion have also been studied in conjunction with breakup to provide a holistic view of droplet dynamics in these types of pressure atomizers.

\section{EXPERIMENTAL SETUP}

The schematic diagram of the experimental setup utilized in this study is shown in Figure 1. The system uses an autoclave (injection pressures up to $7.5 \mathrm{MPa}$ ), test nozzles, and a three axis transverse system which precisely controlled the placement of the nozzle (25.4 $\mu \mathrm{m}$ increments) with respect to the diagnostic systems. This work utilizes only PDPA and shadowgraph techniques to quantify the velocity, droplet shapes, and diameter distributions. The shadowgraph technique used a $532 \mathrm{~nm}$ (maximum energy $70 \mathrm{~mJ} /$ pulse) dual pulsed Nd-YAG laser $(2 \mathrm{~mm}$ beam diameter) synchronized with an interline CCD camera (pixel resolution of $1376 \times 1040$ ). A $90^{\circ}$ turning mirror and a circular diffuser $\left(20^{\circ}\right)$ were used to convert the $2 \mathrm{~mm}$ beam into a diffused mode to provide adequate backlighting for the spray. The camera along with a Navitar zoom lens was placed in front of the spray. The setup with the zoom lens allowed a viewing window of $0.9 \mathrm{~mm} \times 0.9 \mathrm{~mm}$ with a depth of field of $\sim 200 \mu \mathrm{m}$. For shadowgraph, the spatial resolution achieved through this camera-lens setup was around $1 \mu \mathrm{m} /$ pixel.

The PDPA system consisted of a $632 \mathrm{~nm}$ He-Ne laser with three adjoining photo-multiplier tube receiver set at the appropriate receiving angle of $70^{\circ}$ based on the Brewster effect on the surface of the water droplet for first order refraction angle (Figure 1).

\section{RESULTS}

The study used two pressure-swirl hollow cone hydraulic nozzles supplied by Parker Hannifin. The nozzles are classified in Table I.

For droplet diameter distribution along axial and radial direction, results are reported in terms of Reynolds number. Reynolds number can be determined using a velocity scale based on the pressure

TABLE I. Nozzle properties.

\begin{tabular}{lcc}
\hline \hline Nozzle & Flow number $\left(\right.$ pound mass $\left./ \mathrm{h} / \mathrm{psi}^{0.5}\right)$ & Orifice diameter $(\mathrm{mm})$ \\
\hline $\mathrm{N} 1$ & 0.4 & 0.3 \\
$\mathrm{~N} 2$ & 1.7 & 0.5 \\
\hline \hline
\end{tabular}


drop, ${ }^{25} U_{\text {scale }}=\sqrt{\frac{2 \Delta P}{\rho_{l}}} ; \operatorname{Re}_{p}=\frac{\rho_{l} . D}{\mu_{l}} \sqrt{\frac{2 . \Delta P}{\rho_{l}}}$, where $D$ is the nozzle orifice diameter, $\mu_{l}$ is the liquid viscosity, $\rho_{l}$ is the liquid density, and $\Delta P$ is the pressure differential.

To fully characterize the flow and understand the spray dynamics for these pressure-swirl induced hydraulic nozzles, axial and radial measurements were made throughout the spray at various Reynolds numbers (injection pressures) and injector nozzles. PDPA was the principal optical technique used for measuring droplet diameter and velocity while flow visualization for droplet breakup and coalescence was performed using shadowgraph. Note that the one-dimensional PDPA is capable of making only axial component of velocity measurements. This would have an effect on the velocity values especially towards the outer periphery of the spray where the radial velocity component is stronger than inner locations. However, the physics that governs the flow can be estimated even with the availability of only axial velocity components. The spray cone angle measurement (not shown here) reaches a constant value of $78^{\circ}$ at $\Delta P=1.4 \mathrm{MPa}$ for Nozzle N1 and $100^{\circ}$ for Nozzle N2.

\section{A. Hollow cone spray dynamics: Measurements and observations}

\section{Axial measurements}

Even though the nozzles being studied are hollow cones, a significant number of drops are distributed throughout the core of the spray (due to the centrifugal force associated with the pressure swirl) that causes breakup and coalescence (to be discussed later). Thus, axial measurements are still needed in order to understand the spray dynamics of these types of injector nozzles. First, results from PDPA are presented for 10000 spherically validated samples. The variation of arithmetic mean diameter along the centerline of the spray cone for different Reynolds numbers and injector nozzles is reported in Figures 2(a) and 2(b), respectively. For all Reynolds numbers (both nozzles), the droplet diameter decreases along the centerline initially and then increases away from the nozzle tip. Such a behavior of droplet diameter has also been reported by Kim et al. ${ }^{8}$ However, the current measurements are reported for a larger range of axial distance from the nozzle exit than the ones previously reported. The trend suggests the presence of the first flow regime, namely, droplet deformation and breakup, where the droplet diameter decreases, and the regime of coalescence, where droplet diameter increases with axial direction. This is also illustrated in Figure 3, where shadowgraph images are acquired to illustrate the breakup regimes including the primary breakup regime (liquid sheet breakup).

The liquid discharges out of the spray nozzle in the form of films, which break up due to hydrodynamic instability in the form of ligaments (Figure 3). This regime is primarily characterized by formation of ligaments and larger droplets through primary breakup, which could not be explored due to PDPA system's limitation of achieving high spherical validation in the zone of liquid film. The larger droplets formed through primary breakup undergo cascading secondary atomization due to aerodynamic/shear instabilities generating smaller droplets (Figure 3). Thus, the droplet diameter in this zone decreases rapidly as we move away from the nozzle. In addition to secondary atomization,
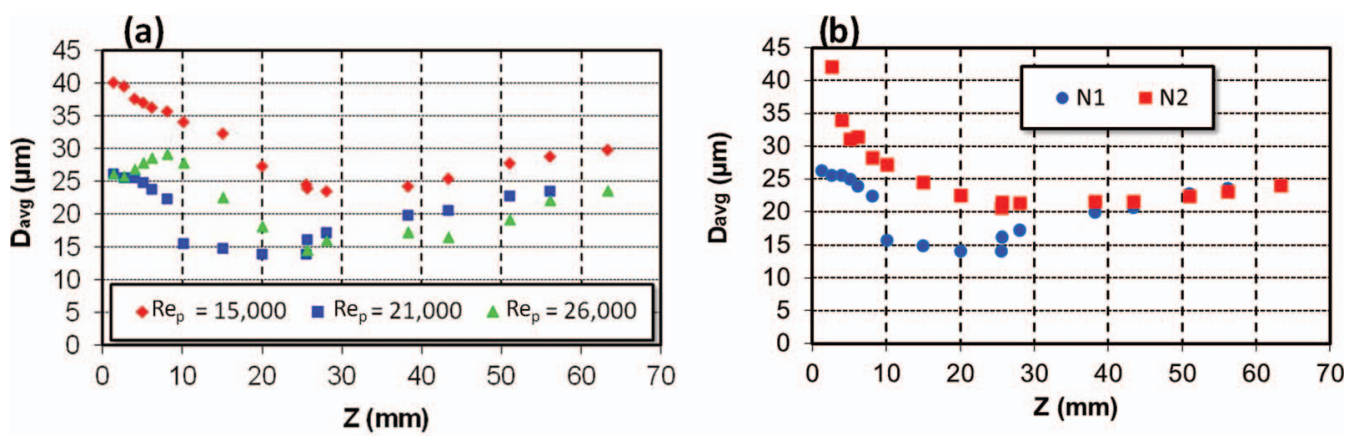

FIG. 2. Diameter measurements along the centerline using PDPA: (a) Reynolds number effects for nozzle N1, (b) nozzle effects at $\operatorname{Re}_{\mathrm{p}}=21000$. 


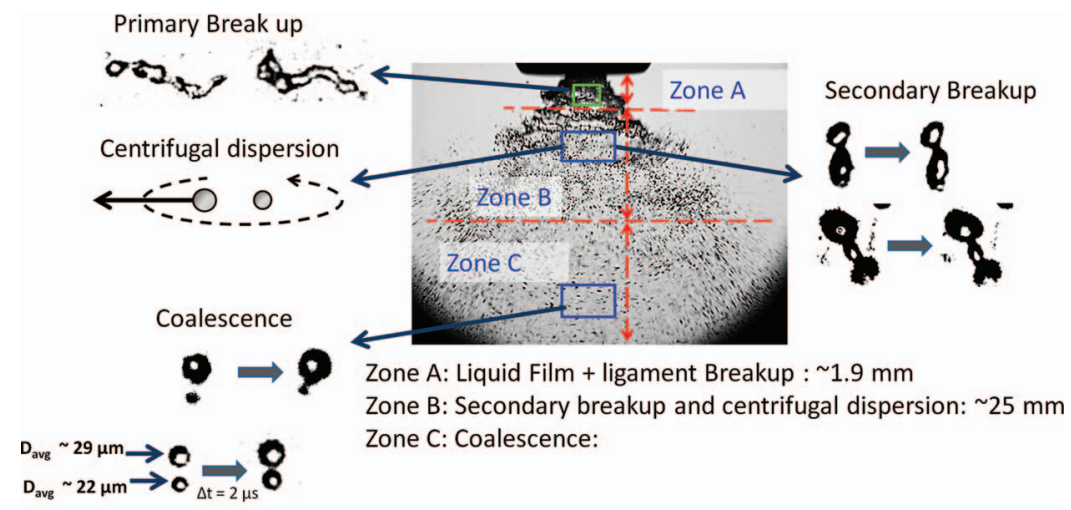

FIG. 3. Representative spray profile for nozzle $\mathrm{N} 2$ at $\mathrm{Re}_{\mathrm{P}}=21000$ indicating typical values of lengths for spray zones with dominant mechanism: Zone A: primary breakup; Zone B: secondary breakup and centrifugal dispersion; Zone C: coalescence.

droplet coalescence also becomes important along the axis. During this process, smaller droplets collide, form larger droplets, and lose their momentum due to drag. Thus, the droplet diameter starts increasing away from the nozzle. As the droplets become larger and slower, the probability of subsequent droplet-droplet collision increases, resulting in further coalescence. In addition to this, the centrifugal effect due to pressure swirls leads to carrying the heavier droplets towards the outer perimeter of the spray cone depleting larger droplets from the center. To understand these competing processes and to identify the transition points we explore the physics governing these mechanisms through various measurements in later sections.

The transition points from one regime to the other regime are observed to shift slightly towards the nozzle as Reynolds number increases (Figure 2(a)). With an increase in Reynolds number, the spray angle and mass flow rate increase while the penetration depth decreases. High Reynolds number leads to increase in drag force on the droplets which subsequently reduces the momentum causing a higher probability of coalescence, resulting in shorter primary and secondary breakup regimes.

At very high Reynolds numbers $\left(\operatorname{Re}_{\mathrm{p}}>25000\right)$, one can observe deviation from this droplet diameter trend (Figure 2(a)). The spray induces a strong recirculation zone close to the nozzle exit. This results in a bimodal distribution of droplet velocities with peaks at $\sim 10 \mathrm{~m} / \mathrm{s}$ and $\sim 45 \mathrm{~m} / \mathrm{s}$, respectively (Figure 4). The recirculation zone causes a higher probability of droplet coalescence at a relatively smaller distance from the nozzle (Figure 4(a)). This early coalescence is further corroborated by the increase in diameter as shown in Figure 2(a). Beyond a certain distance from the nozzle tip, i.e., $\sim 10 \mathrm{~mm}$, the recirculation becomes weak and the smaller droplet velocity peak disappears. Once the droplets leave the recirculation zone, they continue on the traditional breakup path resulting in a similar diameter trend as observed at lower pressures. This can be seen clearly in Figure 4(b), where the droplet diameter exhibits a bimodal distribution with the second peak shifting to lower diameter values, indicating secondary breakup. It was also observed that the recirculation zone, where the droplet velocity is characterized by the bimodal distribution, becomes shorter as the Reynolds number is increased beyond $\operatorname{Re}_{\mathrm{p}}=25000$. In general, the droplet diameter is likely to display a similar profile when the cone angle reaches an asymptotic value at higher Reynolds number. However, the length of the recirculation zone near the nozzle exit alters with Reynolds number even beyond this point. For nearly the same Reynolds number, when the nozzle diameter was increased (Nozzle N2), the transition zone from secondary breakup to coalescence is seen to shift away from the nozzle exit compared to Nozzle N1 (Figure 2(b)). This indicates that this transition zone is also dependent on the nozzle geometry in addition to the Reynolds number. Though the trends are very different initially, both nozzles exhibit similar values of droplet diameter far downstream of the nozzle. The initial difference is associated with the droplet distribution at the nozzle exit where the larger nozzle N2 produces larger diameter droplets. This is due to the higher mass flux, which in turn, produces a thicker liquid film that subsequently results in larger ligaments which finally 

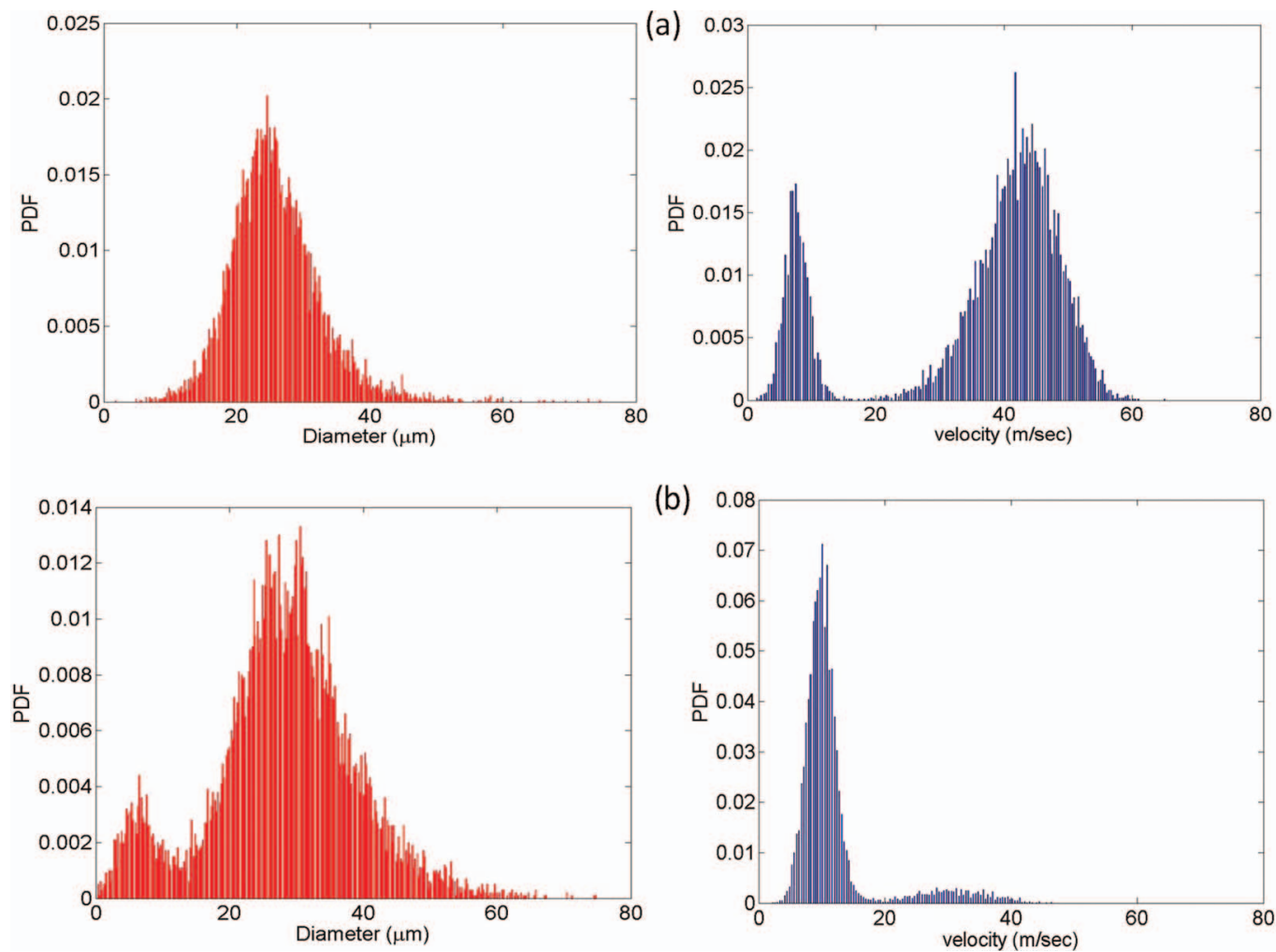

FIG. 4. Histogram plots at $\mathrm{Re}_{\mathrm{p}}=26000$ for nozzle N1: (a) $4 \mathrm{~mm}$ axial location and (b) $10 \mathrm{~mm}$ axial location.

shear off to form larger droplets compared to Nozzle N1. However, further downstream, the droplet diameters for both nozzles approach similar diameter values. This similarity is characterized from the swirl aspect of these nozzles. For the larger nozzle (N2) the spray cone angle is larger; thus, the majority of the droplets are swept to the periphery during the initial stages of breakup creating a sparsely populated core. Therefore, at downstream locations, the droplets exhibit a lower probability of coalescence particularly at the center. Details of the theory and mechanisms of droplet breakup and coalescence can be found later in the text (Sec. III B).

Figure 5 shows the effects of Reynolds number and nozzle diameter on droplet velocity along the centerline. The results show that the droplet velocity decreases rapidly within the first $25.4 \mathrm{~mm}$ from the tip of the nozzle and then decreases at a slower rate until it reaches $\sim 5 \mathrm{~m} / \mathrm{s}$ for both nozzles and Reynolds numbers. These results are in good agreement with the literature. ${ }^{1}$ This can be observed for both nozzles and all Reynolds number. It is important to note that as the Reynolds
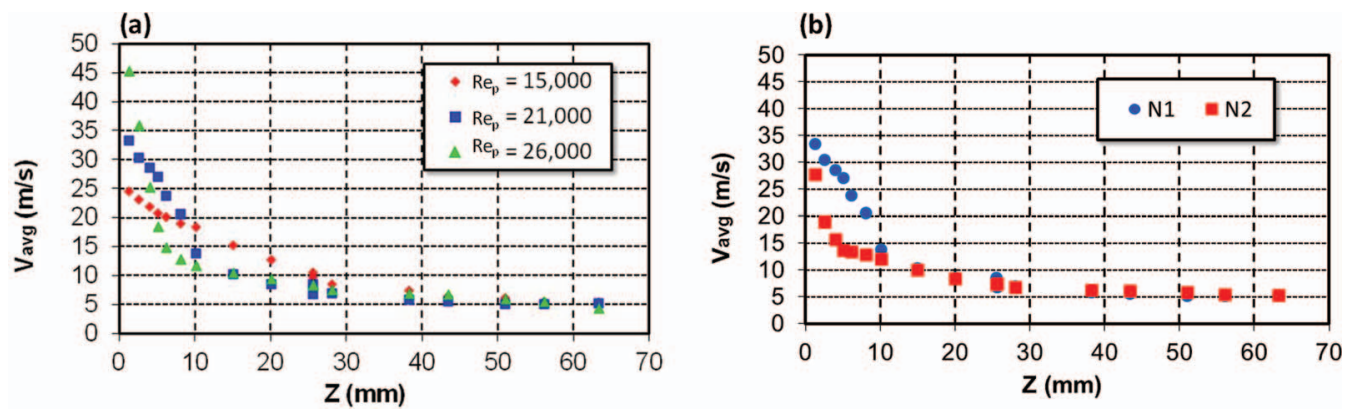

FIG. 5. Velocity measurements along the centerline: (a) Reynolds number effects for nozzle N1; (b) nozzle effects at $\operatorname{Re}_{\mathrm{p}}=21000$. 
number increases, the slope of the velocity within the first $25.4 \mathrm{~mm}$ becomes steeper due to the larger drag force (larger back pressure). The sharp drop seen for $\mathrm{Re}_{\mathrm{p}}=26000$ is associated with the bimodal distribution which dramatically lowers the average velocity of the droplets. For larger nozzle (N2), at locations close to the nozzle, the velocity values are much smaller compared to the smaller nozzle (N1) due to higher initial momentum flux.

\section{Radial measurements}

As described in Sec. III A 1, for full characterization of the flow with the complex nature of hollow cone sprays, radial measurements were needed at various Reynolds numbers (injection pressures) and injector nozzles. Figures 6(a)-6(c) show the data rate, average diameter, and velocity as a function of radial location for the small nozzle (N1). Measurements were made at an axial location of $10 \mathrm{~mm}$ from the nozzle and $\mathrm{Re}_{\mathrm{p}}=9500$ as shown in Figure 6(d). It can be noticed that there exists a linear decay in data rate where the maximum occurs in the core and the minimum at the periphery. Data rate represents the population density of droplets. In a hollow cone spray, bulk of the droplets is supposed to remain in the outer regime of the spray cone. Thus, the trend is opposite to what is expected. At this particular Reynolds number, the spray cone angle is very small $\left(\theta=25^{\circ}\right)$. Low spray angle restricts the liquid film to spread in radial direction. Moreover, the liquid film thickness being comparable to the radius of the nozzle, the film around the nozzle intersects across the diameter of the nozzle near the nozzle tip (or low axial distances). Thus, majority of the droplets will be entrapped in the core causing the data rate to be highest in the core and lowest at the edge. Due to this overlap, the diameter and velocity start out high in the core and reduces linearly radially outwards as observed in Figure 6. The swirling flow or the tangential component of the flow in a pressure-swirl nozzle helps to spread the droplets towards the outer periphery and larger droplets are more sensitive to this radial motion due to higher centrifugal force. As the swirling strength is weak for lower Reynolds numbers, the majority of the droplets are accumulated in the core. This also results in low probability of coalescence in the radial direction allowing the droplets to continue
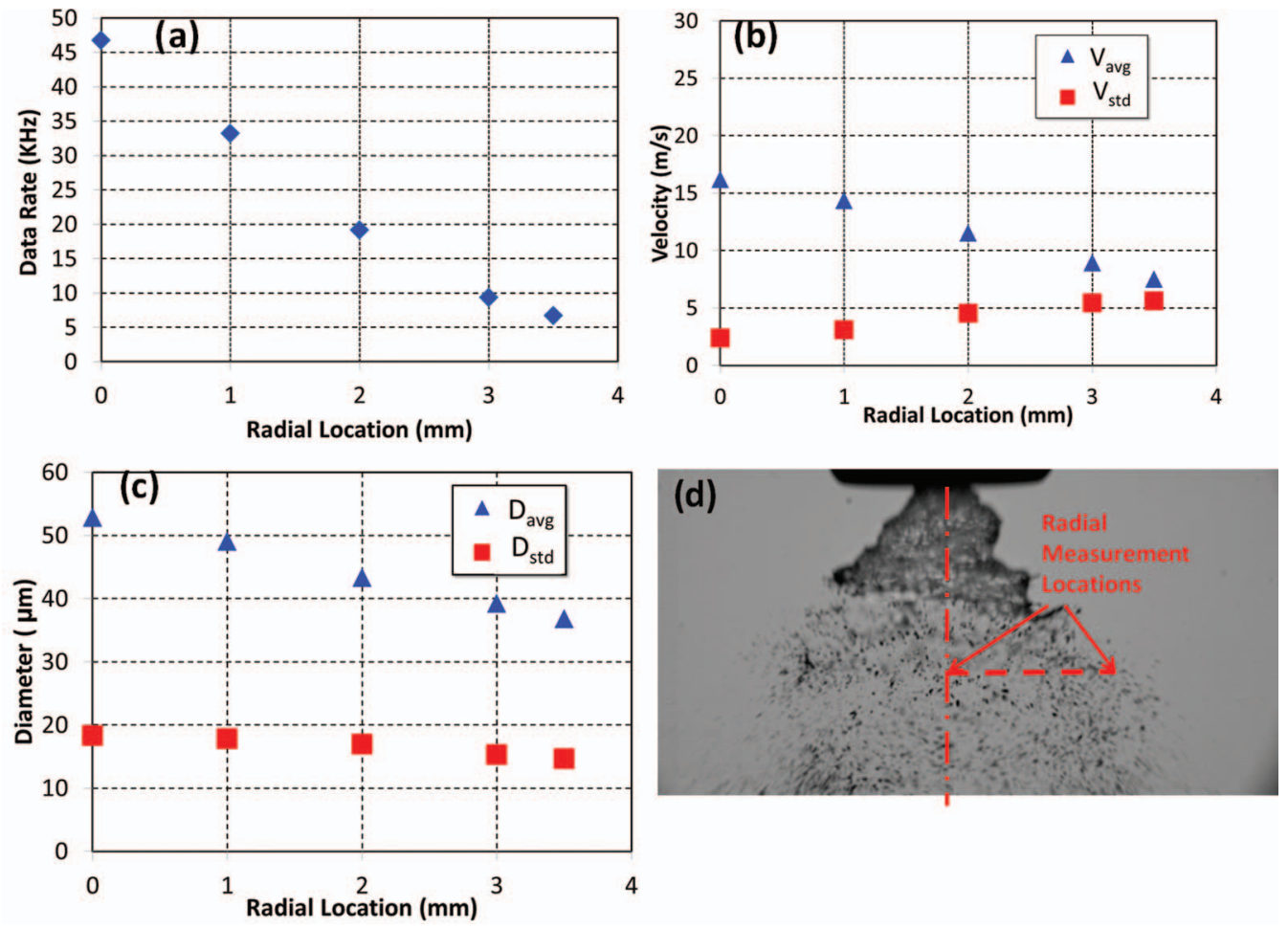

FIG. 6. Radial measurements at $Z=10 \mathrm{~mm}, \mathrm{Re}_{\mathrm{p}}=9500$, nozzle $\mathrm{N} 1, \theta=25^{\circ}$ : (a) data rate, (b) velocity profile, (c) diameter profile, (d) picture of spray profile for nozzle $\mathrm{N} 2$ at $\mathrm{Re}_{\mathrm{P}}=15000$. 

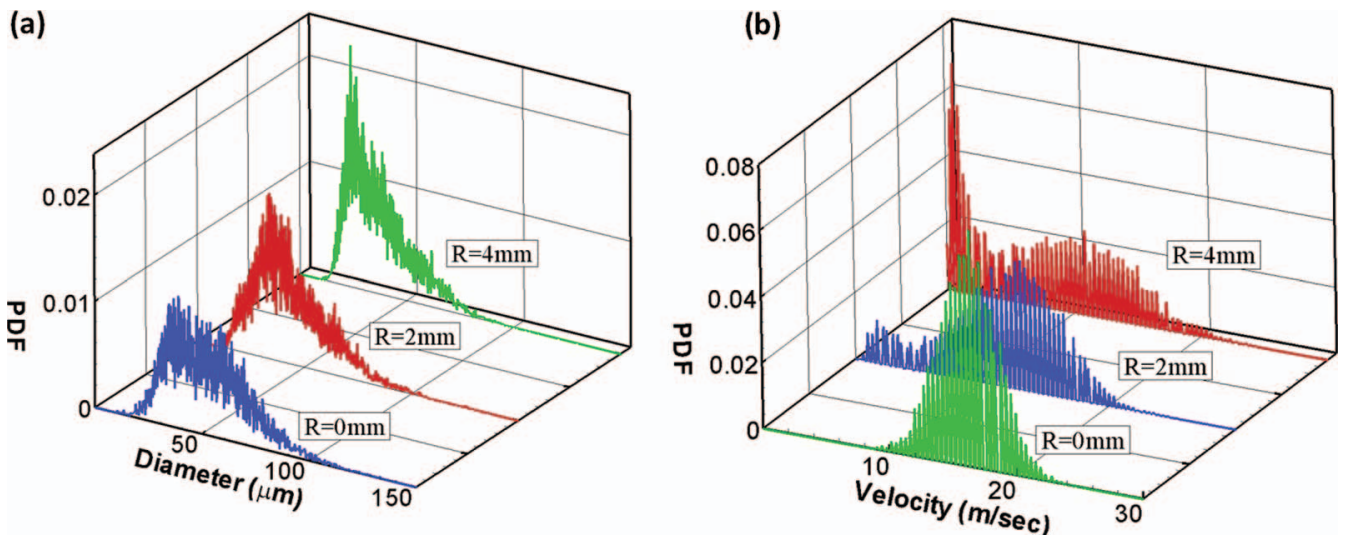

FIG. 7. Radial histograms at $Z=10 \mathrm{~mm}, \mathrm{Re}_{\mathrm{p}}=9500$, nozzle $\mathrm{N} 1, \theta=25^{\circ}$ : (a) diameter and (b) velocity.

on the principal droplet breakup cycle. This can clearly be seen in Figure 7(a) where the diameter histogram clearly shows a shift in the peak location with change in radial location. In Figure 7(b), we also observe the introduction of a second peak for the velocity histogram which also confirms breakup. Applying conservation of momentum during the droplet breakup process, it can be shown that the velocity of the daughter droplets is lower than the parent droplet thereby introducing the second peak in the velocity histogram (Figure 7(b)). The bimodal nature of the velocity histogram at the periphery of the spray is also reflected in the form of a lower mean $\left(\mathrm{V}_{\text {avg }}\right)$ and a higher standard deviation $\left(\mathrm{V}_{\text {std }}\right)$ as shown in Figure 6(b).

Figure 8 shows the change in the data rate, velocity, and diameter of the droplets with radius at the axial location $63 \mathrm{~mm}$ for the small nozzle (N1) and $\mathrm{Re}_{\mathrm{p}}=9500$. Although the liquid film at this particular location does not intersect, the droplet population still remains high at the center core of the spray. The droplets which were trapped at the core near the nozzle exit fail to reach the periphery even at higher axial distances due to weak swirl at low Reynolds number. Thus, the data rate will still be high towards the center and low towards the periphery. However, far from the nozzle exit at $63 \mathrm{~mm}$, the diameter increases towards the periphery (Figure 8(c)) due to centrifugal effect and higher coalescence probability. As reported in the previous paragraph, droplets in the outer periphery experience a strong coalescence causing increase in diameter. This is also indicated by the decrease in standard deviation which shows a decreasing profile, implying collision of smaller droplets to form larger droplets resulting in a decrease in the half-width of the histogram. This is also evident in the velocity plot where the values decrease radially which is caused by newly formed droplets (due to collisions) which experience a higher drag force. Since the standard deviation for the velocity component is very similar throughout all radial locations, uniform droplet coalescence is to be expected.

Figure 9 displays the spray characteristics for the same nozzle (N1) at higher Reynolds number $\left(\operatorname{Re}_{\mathrm{p}}=21000\right)$. For this Reynolds number, the spray angle is much wider $\left(\theta=74^{\circ}\right)$; therefore, the
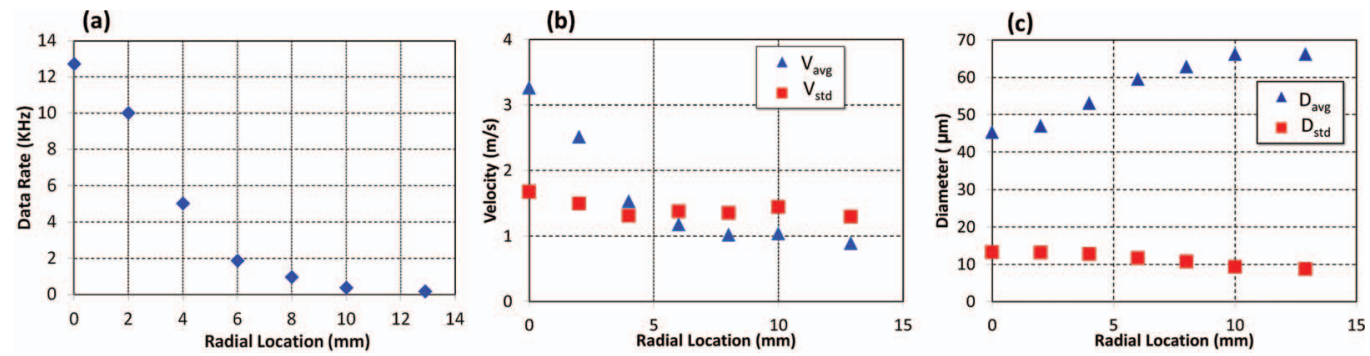

FIG. 8. Radial measurements at $Z=63 \mathrm{~mm}, \mathrm{Re}_{\mathrm{p}}=9500$, nozzle $\mathrm{N} 1, \theta=25^{\circ}$ : (a) data rate, (b) velocity profile, (c) diameter profile. 

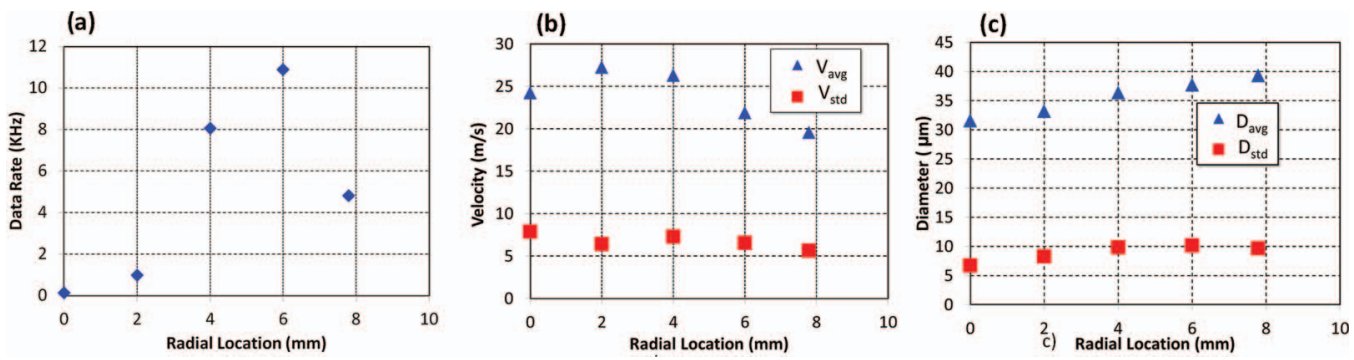

FIG. 9. Radial measurements at $\mathrm{Z}=10 \mathrm{~mm}, \mathrm{Re}_{\mathrm{p}}=21000$, nozzle $\mathrm{N} 1, \theta=75^{\circ}$ : (a) data rate, (b) velocity profile, (c) diameter profile

liquid film will not intersect near the nozzle exit. This causes the data rate, which also characterizes droplet density at different locations, to follow what is traditionally expected from a hollow cone nozzle. The larger spray angle causes the data rate to be lower at the core and larger towards the periphery. Figure 9 (a) shows that the data rate increases radially reaching a maximum value at a radial location of $6 \mathrm{~mm}$. Beyond this radial distance, the data rate starts decreasing as the measurement point approaches the outer extreme of the spray. The location of this outer extreme where the data rate reduces to $1 \%$ of the maximum data rate is close to the estimated value based on the spray angle. The average velocity is seen to increase mildly before it starts decreasing with radial locations. With high data rate (or droplet population towards the outer radius), droplets are more prone to coalescence resulting in higher average droplet diameter (Figure 9(c)). Besides coalescence, the centrifugal action also helps the heavier droplets to move towards outer periphery. Larger droplets move slowly to maintain the same momentum, resulting in reduction in average velocity $\left(\mathrm{V}_{\text {avg }}\right)$ as shown in Figure 9(b).

The data rate at a further downstream axial location of $63 \mathrm{~mm}$ (Figure 10(a)) shows a similar increase in date rate with radial distance. However, once it reaches a maximum value at radial location $6 \mathrm{~mm}$, it reduces rather slowly compared to the axial distance, $\mathrm{Z}=10 \mathrm{~mm}$. This indicates that as the spray moves axially downstream the thickness of the zone, where the majority of the droplets occur, also increases. Thus, a comparatively larger zone in the radial direction contains moderate to high data rate or droplet population. Stronger swirl or tangential velocity brings the droplets towards the periphery inducing coalescence. Coalescence in the outer radius along with the centrifugal effect increases the average droplet diameter while the axial velocity becomes lower (Figures 10(b) and 10(c)). Radial data for N2 nozzle (not reported here) shows similar trends as the N1 nozzle.

\section{B. Dynamics of film breakup, secondary atomization, and coalescence}

All the results presented for axial and radial measurements for several Reynolds numbers indicate that droplet dynamics is affected by the simultaneous presence of breakup (primary and
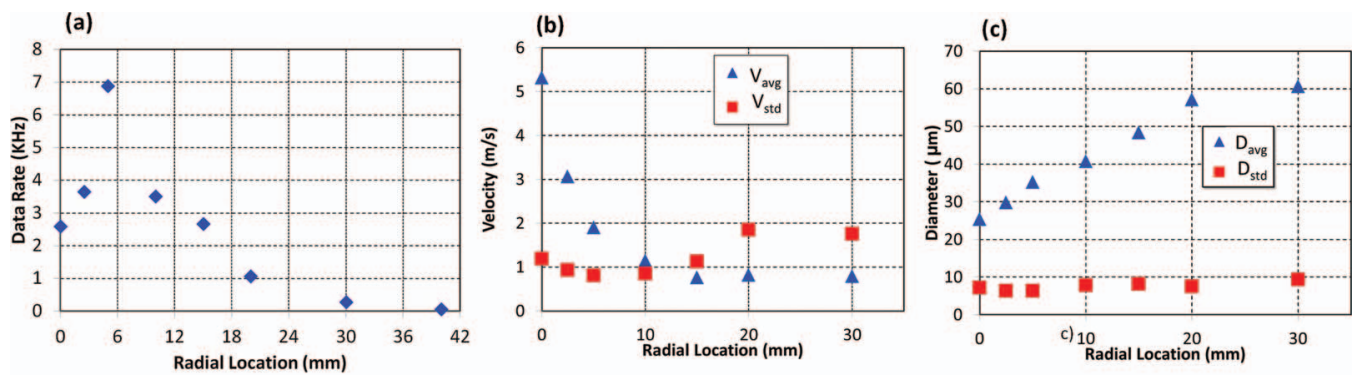

FIG. 10. Radial measurements at $\mathrm{Z}=63 \mathrm{~mm}, \mathrm{Re}_{\mathrm{p}}=21000$, nozzle N1, $\theta=74$ : (a) data rate, (b) velocity profile, (c) diameter profile. 
secondary), coalescence, and strong centrifugal action of the swirl. The potency of these mechanisms varies in different zones of the spray, where these mechanisms are functions of the Weber number, Reynolds number, and nozzle geometry. In order to assimilate and reconcile the observations reported in Figures 2-10, under a consistent framework, each of the breakup, coalescence, and centrifugal dispersion mechanisms are analyzed in detail.

\section{Breakup of droplets}

In a hollow cone pressure swirl nozzle, droplet generation takes place through a series of primary and secondary breakup processes ${ }^{2,3}$ as explained previously. The pressure differential across the nozzle and the swirl inside the nozzle injects a liquid film through the orifice. The relative velocity between this liquid film and the ambient gas phase (air in current experiment) induces an instability which grows as the liquid film moves away from the nozzle, and eventually breaks up in the form of ligaments. These unstable ligaments, in general, go through further atomization, and generate droplets. These droplets, in most cases, exhibit secondary atomization generating smaller daughter droplets due to aerodynamic breakup as discussed briefly in Sec. I. The dynamics of these breakup processes of the liquid film, which is schematically shown in Figure 11, occurs within a very small region next to the nozzle exit. This is fundamentally important as these processes control the droplet diameter downstream of the nozzle. Senecal et $a .^{3}$ modeled breakup of two-dimensional liquid films. They showed that based on the Weber number (We), the linear instability in liquid films can be categorized into long wavelength and short wavelength with a critical Weber number ( $\left.W e_{c}\right)$ of 27/16. The long wavelength instability occurs for $W e<W e_{c}$ and the short wavelength occurs for $W e>W e_{c}$. Here, the Weber number is defined as $W e=\frac{\rho_{g} U_{l}^{2} t}{\sigma}$, where $\rho_{g}$ is the gas phase density, $U_{l}$ is the liquid film velocity, $t$ is half of film thickness ( $t=h / 2, h$ : film thickness), and $\sigma$ is the surface tension of the liquid. Although this analysis has been done for 2D liquid films, in general, we extended it for a hollow cone liquid film, which can be simplified as a two-dimensional film. At the nozzle exit, the liquid film forms a rim-like structure, which can be unwrapped to form a two-dimensional sheet. ${ }^{4}$

In the current experiment, the velocity of the liquid film is given by $U_{l}=k U_{\text {scale }}$, where $U_{\text {scale }}$ is the velocity scale defined earlier and $k$ is a multiplying factor. Actual definition of " $k$," a scale for estimating the liquid film velocity, was given by Lefebvre, ${ }^{2}$ which depends on nozzle design and injection pressure. However, later Senecal et $a .^{3}{ }^{3}$ and Schmidt et al. ${ }^{4}$ found that Lefebvre's correlation led to unphysical values, such as velocity coefficient being greater than 1 . They further argued that one could consider the swirl ports to be nozzles, and the equation, $U_{\text {scale }}=k \sqrt{\frac{2 . \Delta P}{\rho_{l}}}$

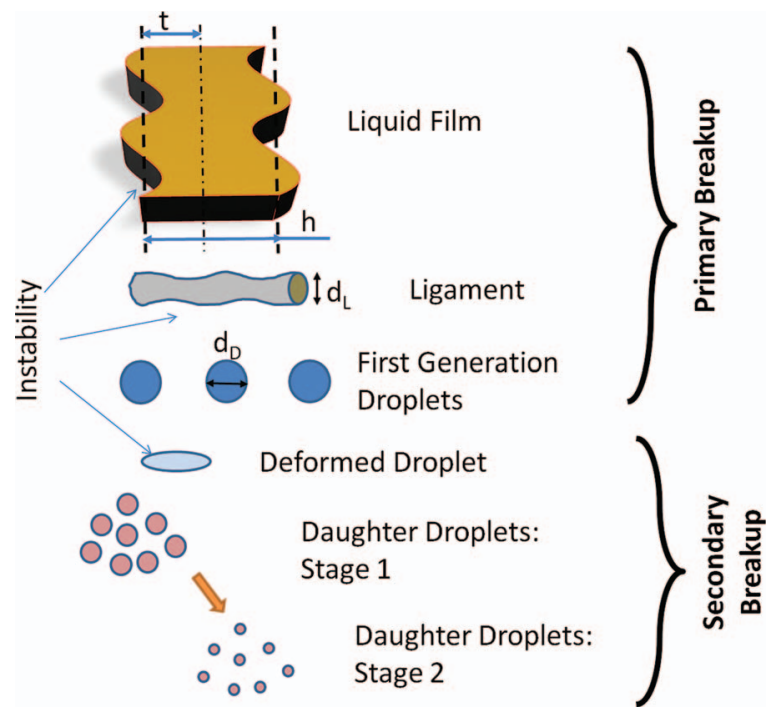

FIG. 11. Different stages of liquid film breakup and droplet formation. 
represented an expression for the coefficient of discharge for the swirl-ports with the assumption that the majority of the pressure drop occurred across the injector ports. The discharge coefficient $\left(C_{d}\right)$ for single phase nozzles with sharp inlet corners and an $L / D$ of 4 is typically 0.78 or less as reported by Lichtarowicz et al. ${ }^{26}$ and under cavitation it can be as low as 0.61 . Hence, 0.78 should be a practical upper bound for $k$. Considering $10 \%$ loss in discharge coefficient due to other momentum losses across the injector, Senecal et $_{\text {al. }}{ }^{3}$ arrived at the value 0.7 as the upper limit, the same value used in this study. Furthermore, there are some physical considerations which limit the value of $k$. It can never be more that 1 (value less than unity) following energy conservation, it must allow sufficient mass flow with non-negative air-core thickness. To satisfy all these conditions, Senecal et al. ${ }^{3}$ and Schmidt et al. ${ }^{4}$ applied the condition of $k=\max \left[0.7, \frac{4 \dot{m}}{\pi d_{0}^{2} \rho \cos (\theta)} \sqrt{\frac{\rho}{2 . \Delta P}}\right]$, where $\dot{m}$ is the mass flow rate and $\theta$ is the half cone angle. In our experiments, we found this value to be always 0.7. As the length of this film zone is of the order of a few millimeters, one can assume that the bulk velocity of the liquid film remains constant and takes on a value of the velocity scale, $U_{l}$. From the mass flow rate of the nozzle, one can estimate the liquid film thickness at the nozzle tip from the following equation: ${ }^{23}$

$$
h=3.66\left(\frac{\dot{m} D \mu_{l}}{\Delta P \rho_{l}}\right)^{0.25},
$$

where $\rho_{l}$ is the liquid density, $\mu_{l}$ is the liquid viscosity, $h(=2 t)$ is the film thickness, $\Delta P$ is the injection pressure, and $D$ is the nozzle orifice diameter. According to the film breakup theory, the instability grows very rapidly in a sinusoidal manner. The wavenumber of this instability can be expressed as

$$
\left.\begin{array}{l}
K_{s} t=\frac{W e}{2} \text { (for long wavelength) } \\
K_{s} t=\frac{2 W e}{3} \text { (for short wavelength) }
\end{array}\right\} .
$$

Using the relation between wavelength and wave number $\left(K_{s}=2 \pi / \lambda\right)$ one can write

$$
\left.\begin{array}{l}
\frac{\lambda}{h}=\frac{2 \pi}{W e} \text { (for long wavelength) } \\
\frac{\lambda}{h}=\frac{3 \pi}{2 W e} \text { (for short wavelength) }
\end{array}\right\} .
$$

To determine the most unstable wavelength experimentally, shadowgraph technique was used. As shown in Figure 12, pixel lengths were measured for each frame to obtain the wavelength for different Reynolds numbers for each nozzle. Most unstable wavelength $(\lambda)$ denotes the distance from the peak of one wave to another wave just before the breakup of the liquid film (Figure 12). Typically, wavelengths were obtained by averaging 20-30 images as shown in Figure 12, where the standard
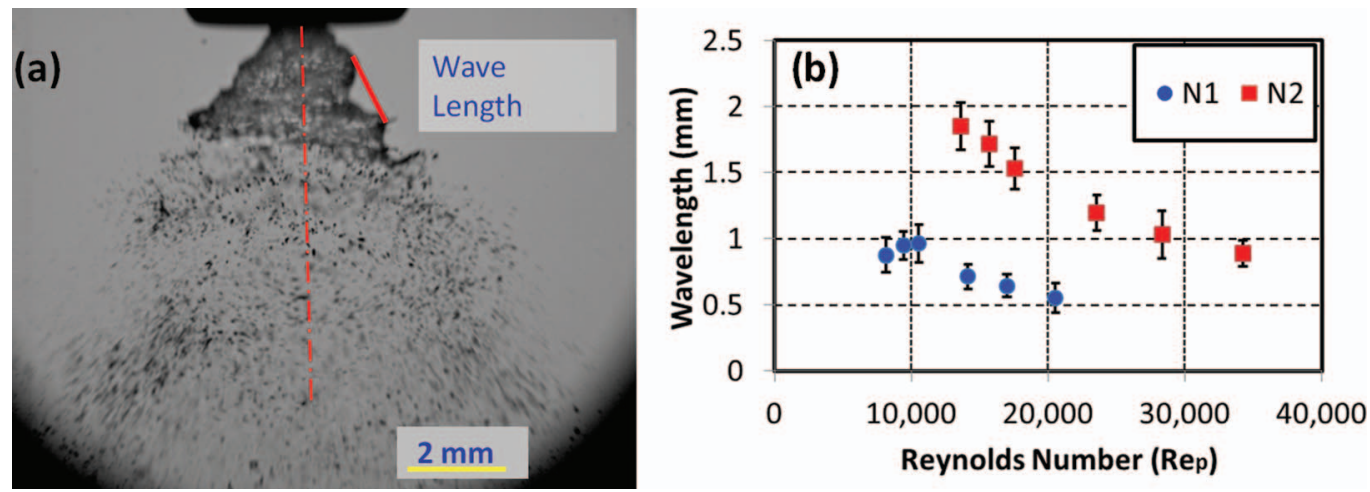

FIG. 12. Change in most unstable wavelength with Reynolds number; (a) representative picture of the spray profile for nozzle $\mathrm{N} 2$ at $\mathrm{Re}_{\mathrm{P}}=15000$. (b) Wavelength profile. 


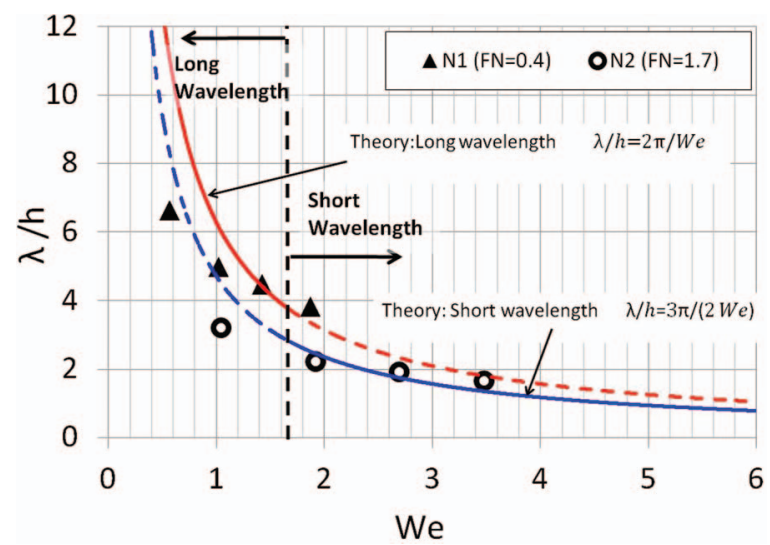

FIG. 13. Comparison of most unstable wavelengths from breakup models with experimental results.

deviations are used as the uncertainty. From Figure 12(b), one can notice that the trends for the maximum wavelength are not significantly different for the two nozzles. At low Reynolds numbers, the instabilities in the liquid film for the small nozzle (N1) converge into a sharp point with small amplitudes and long wavelengths that trigger breakup. With increase in Reynolds number, the flow angle sharply increases, resulting in an outward flow expansion and the instabilities become shorter in wavelengths and larger in amplitude. Thus, the maximum wavelength reduces with increase in Reynolds number. On the other hand, the flow for the larger nozzle (N2) expands outwards for the entire range of Reynolds numbers tested but still a shift between long wavelengths and small amplitudes to short wavelengths and larger amplitudes can be seen. The cone angle does not increase significantly with the Reynolds number for this nozzle. The larger nozzle (N2) experiences this short wavelength type of instability breakup for the majority of Reynolds number tested. Even though the operating pressure range is almost the same for both the nozzles, the maximum wavelength does not match. This is associated with the relation between the film thickness and Weber number as seen in Eq. (3).

If we calculate or estimate the film thickness $(h)$ of the nozzles at different pressures using Eq. (1) and use it to non-dimensionalize the most unstable wavelength, it follows the theoretical plot closely as shown in Figure 13. Considering the critical Weber number, $W e_{c}=27 / 16$, the figure demarcates two zones (based on Weber numbers) where long and short wavelength breakups dominate. The figure also shows the theoretical $\lambda / h$ for long and short wavelengths (Eq. (3)) as shown with red and blue lines, respectively. The experimental data seem to follow the theoretical profile closely. Variations can be attributed to the measurement uncertainty and small oscillations in operating pressure during the experiments.

Once the liquid film disintegrates, it forms ligaments. Theoretically, it is assumed that for short wavelength, one ligament forms per wavelength of the liquid film, and that for long wavelength, two ligaments form per wavelength. From simple mass balance, one can find the diameter of these ligaments for both types of instabilities:

$$
\left.\begin{array}{l}
d_{L}=\sqrt{8 \frac{t}{K_{s}}} \text { (for long wavelength) } \\
d_{L}=\sqrt{16 \frac{t}{K_{s}}} \text { (for short wavelength) }
\end{array}\right\},
$$

where $t$ is the half film thickness and $K_{s}$ is the most unstable wave number. Using Eq. (3) along with the relations, $h=2 t$ and $K_{s}=2 \pi / \lambda$, Eq. (4) can be rewritten as

$$
\left.\begin{array}{rl}
d_{L} & =\sqrt{4 \frac{h^{2}}{W e}} \text { (for long wavelength) } \\
d_{L} & =\sqrt{6 \frac{h^{2}}{W e}} \text { (for short wavelength) }
\end{array}\right\} .
$$



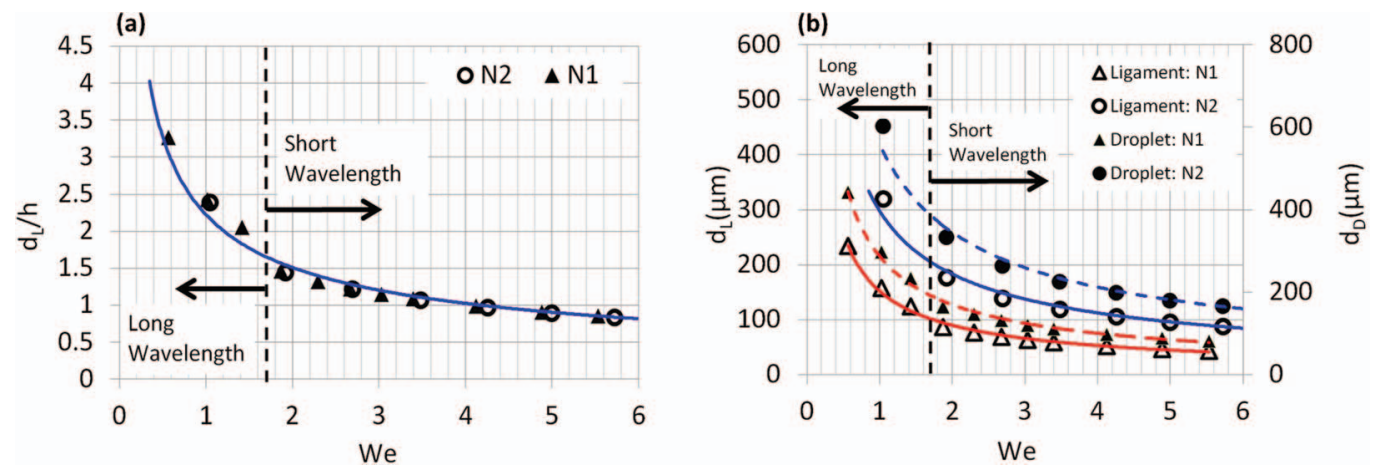

FIG. 14. (a) Non-dimensional ligament diameter ( $d_{L}$ is calculated using Eq. (5); $h$ is calculated using Eq. (1)); (b) dimensional diameter of ligament and droplets after primary breakup.

The non-dimensional ligament diameters of both the nozzles are shown in Figure 14(a) as a function of Weber number. Note that as the non-dimensional ligament diameter $\left(d_{L} / h\right)$ is only a function of $W e$, both nozzles follow a single characteristic curve. However, if the dimensional ligament diameters $\left(d_{L}\right)$ are plotted against We, the two nozzles follow two distinct profiles as shown in Figure 14(b). The film thicknesses $(h)$ for the same We are different for two nozzles resulting in two distinct curves for $d_{L}$.

These cylindrical ligaments are not stable in nature. The surface tension force along with viscous dissipation introduces shape oscillation, and the ligament eventually breaks up into small droplets. Rayleigh ${ }^{27}$ introduced the theory of this type of instability and Dombrowski and Johns ${ }^{28}$ showed that most instability in ligaments leads to the results observed by Weber ${ }^{29}$ using capillary instability analysis. A simple model for this instability considers breakup of a cylindrical liquid column (ligament) and the resultant droplet diameter can be estimated by ${ }^{29}$

$$
d_{D}=1.88 d_{L}(1+3 O h)^{1 / 6} .
$$

The Ohnesorge number, $O h$, is defined as $O h=\mu_{l} /\left(\rho_{l} \sigma d_{L}\right)^{1 / 2}$, where $\mu_{l}, \sigma$, and $\rho_{l}$ are liquid viscosity, surface tension, and density, while $d_{L}$ is the diameter of the ligament. Figure 14(b) shows the droplet diameter $d_{D}$ for different Weber numbers for both nozzles. From Eq. (6) it is noted that $d_{D} / d_{L}$ is always greater than 1, also seen in Figure 14(b).

The initial decrease in the average droplet diameter along the $\mathrm{Z}$ axis (Figure 2) can now be explained by secondary breakup of droplets and dispersion of the droplets through centrifugal effect in the spray cone due to pressure swirl. The primary breakup of the liquid sheet explained in the previous paragraph is strong only at axial distances close to the nozzle. The secondary breakup, on the other hand, mainly occurs due to strong aerodynamic force arising from the relative velocity between the droplets and ambient air. This secondary breakup primarily depends on the diameter based Weber number $\left(W e_{D}\right)$ and Ohnesorge number $\left(O h_{D}\right), W e_{D}=\rho_{g} U_{l}^{2} d_{D} / \sigma$ and $O h_{D}=\mu_{l} /\left(\rho_{l} \sigma d_{D}\right)^{1 / 2}$. The secondary atomization can consist of several types of breakup ${ }^{30,31}$ as shown in Table II.

The secondary breakup process is cascading in nature and will continue until the Weber number of the final stable daughter droplets falls below a critical value. At this point, the breakup process terminates and the process of droplet coalescence starts dominating. In the current experiments, $W e_{D}$ is found to be mostly less than 11 when $O h_{D}$ is of the order of $10^{-3}$. This suggests the droplets generated from ligaments should first go through vibrational shape oscillation and eventual breakup. Although with increase in Weber number, the breakup is more likely to occur, there is no criterion for quantifying this probability. Hsiang and Faeth ${ }^{32}$ showed that for $O h_{D}<0.1$ the non-dimensional breakup time is written as

$$
t_{b} / t^{*}=\mathrm{C},
$$

where $\mathrm{C}$ is a constant and approximated as 5.0 based on several experiments with multitude of liquids. Here, the time scale is calculated by $t^{*}=\frac{d_{0} \sqrt{\left(\rho_{l} / \rho_{g}\right)}}{U_{0}}$, where $d_{0}$ is the initial droplet diameter 
TABLE II. Types of secondary breakup.

\begin{tabular}{lc}
\hline \hline Types of breakup & \multicolumn{1}{c}{ Range of $W e_{D}$} \\
\hline $\begin{array}{l}\text { Vibrational deformation of the droplet and breakup } \\
\text { Bag breakup which causes the large droplet first to } \\
\text { deform into a thin disk normal to the flow direction and } \\
\text { then balloons out leading to disintegration }\end{array}$ & $\left(11<W e_{D}<35\right.$ for $\left.O h_{D}<0.1\right)$ \\
$\begin{array}{l}\text { Multimode breakup which is a combination of both bag } \\
\text { type and sheet thinning breakups }\end{array}$ & $\left(35<W h_{D}<80\right.$ for $\left.O h_{D}<0.1\right)$ \\
$\begin{array}{l}\text { Sheet thinning breakup which causes the droplet to deflect } \\
\text { at the periphery of the droplet disk instead of the center } \\
\text { like bag breakup } \\
\text { Catastrophic breakup which is similar to stripping } \\
\text { breakup but more explosive in nature }\end{array}$ & $\left(80<W e_{D}<350\right.$ for $\left.O h_{D}<0.1\right)$, \\
\hline \hline
\end{tabular}

before breakup, $\rho_{l}$ and $\rho_{g}$ are density of the liquid and gas phase, and $U_{0}$ is the droplet velocity. It may be concluded that larger breakup time, $t_{b}$, signifies lower probability of the breakup. If we calculate the probability density functions (PDF) for the $W e_{D}$ and $t_{b}$ for the droplets at different axial locations along the center line of the spray (Figures 15 and 16), we can gain some useful information that helps us understand the droplet diameter profile.

The PDFs of $W e_{D}$ (Figure 15) at different Reynolds number $\left(\operatorname{Re}_{\mathrm{p}}\right)$ for nozzle N1 reveal that most of the droplets have $W e_{D}<0.1$, however, there is a small percentage of droplets at lower $\mathrm{Z}$ locations with $W e_{D} \sim 1$ representing a population which are more prone to breakup. We also notice that the probability of droplets with lower Weber number increases at higher $\mathrm{Z}$ locations, depicting lower probability of breakup (Figure 15). Comparing the breakup time, $t_{b}$, at any Reynolds number, PDF is seen to shift towards higher values with increase in $\mathrm{Z}$ (Figure 16) showing retardation in breakup process at higher axial distances. The maximum $W e_{D}$ shows a steady declining trend
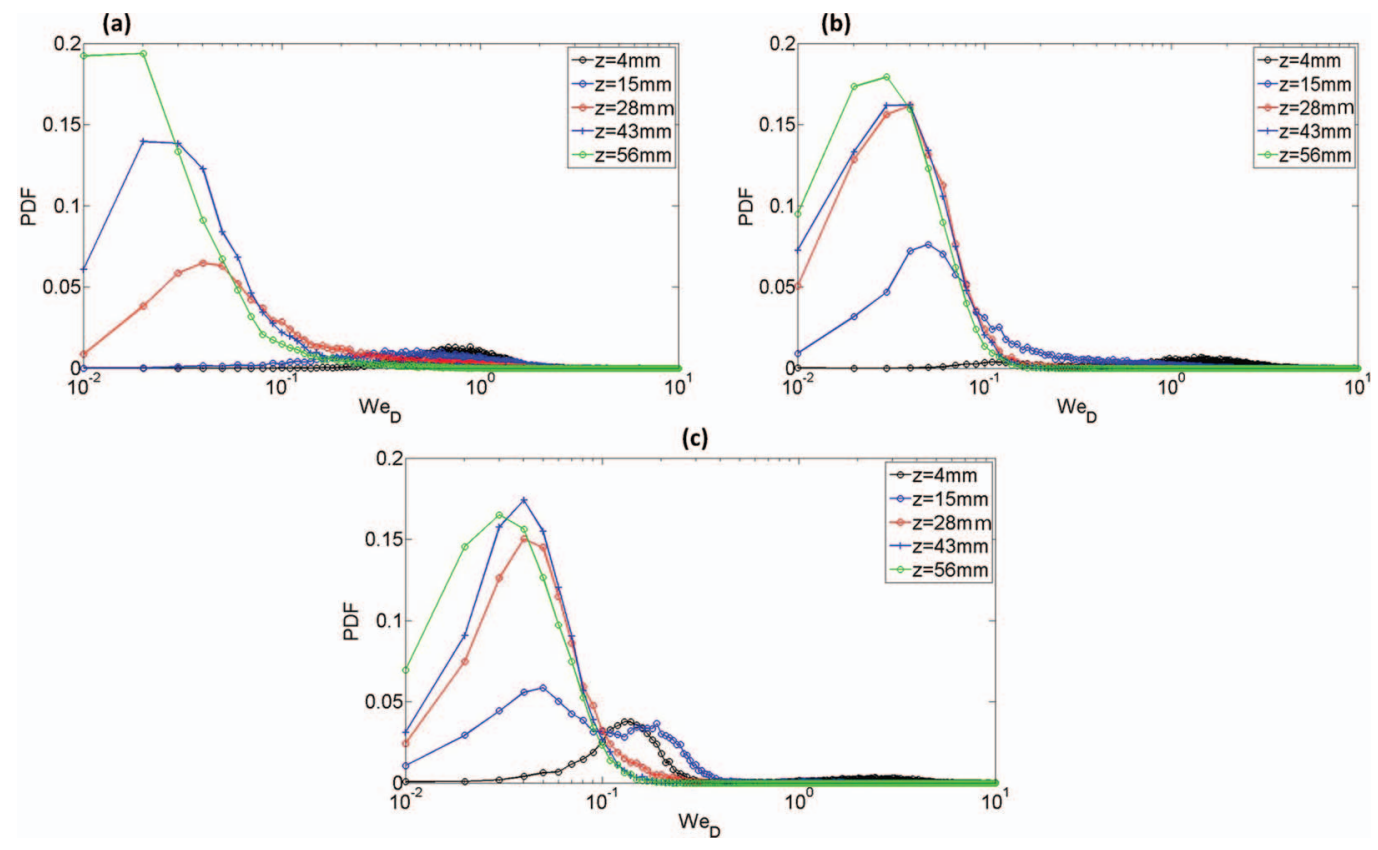

FIG. 15. PDF of $W e_{D}$ at different axial locations along the center line of the spray (Nozzle N1) at different Reynolds number, (a) $\operatorname{Re}_{\mathrm{p}}=10500$, (b) $\operatorname{Re}_{\mathrm{p}}=21000$, (c) $\operatorname{Re}_{\mathrm{p}}=26000$. 
(a)

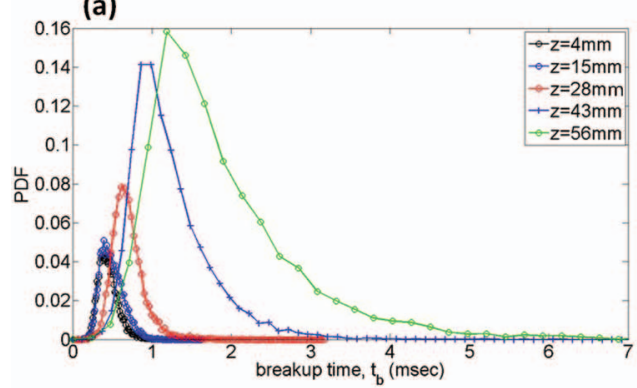

(b)

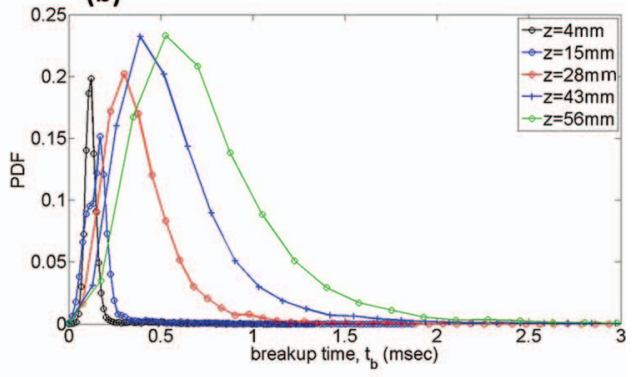

(c)

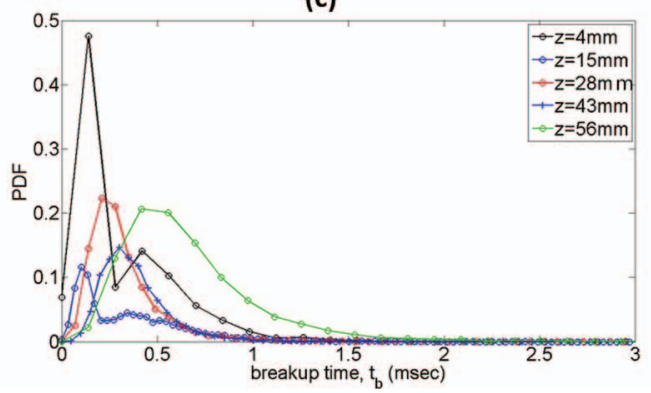

FIG. 16. PDF for the breakup time, $t_{b}$ for the droplets at different axial locations along the center line of the spray (Nozzle N1) at different Reynolds numbers: (a) $\operatorname{Re}_{\mathrm{p}}=10500$, (b) $\operatorname{Re}_{\mathrm{p}}=21000$, (c) $\operatorname{Re}_{\mathrm{p}}=26000$.

(Figure 17(a)) with the $\mathrm{Z}$ location, indicating that the breakup intensity will become weaker with the distance from the nozzle. The maximum Weber number of 4 close to the nozzle exit indicates that secondary breakup is potent in locations immediately downstream of the primary breakup zone. Nozzles N1 and N2 show similar decay rates in terms of maximum Weber number (Figure 17(b)). Nozzle N1 shows a slightly higher maximum Weber number very close to the nozzle exit compared to $\mathrm{N} 2$ nozzle.

If we consider the atomization of the droplets generated by primary breakup, we can estimate the droplet diameters from successive breakups. The secondary breakup process has been experimentally and numerically studied in detail. As mentioned earlier in our experiments the dominant mode of secondary breakup is vibrational deformation/breakup due to aerodynamic forces and thus, droplets generated from ligaments should first go through vibrational shape oscillation and eventual breakup. Among different models of this breakup, Taylor analogy breakup model (TAB) is the most accepted one. ${ }^{31} \mathrm{~A}$ simple spring-damped mass analysis show that the vibrational mode of breakup would result in daughter droplet sizes.

The daughter droplet size can be estimated using energy conservation between the parent and the daughter droplets. This analysis assumes distortion and oscillation in the parent droplet, while
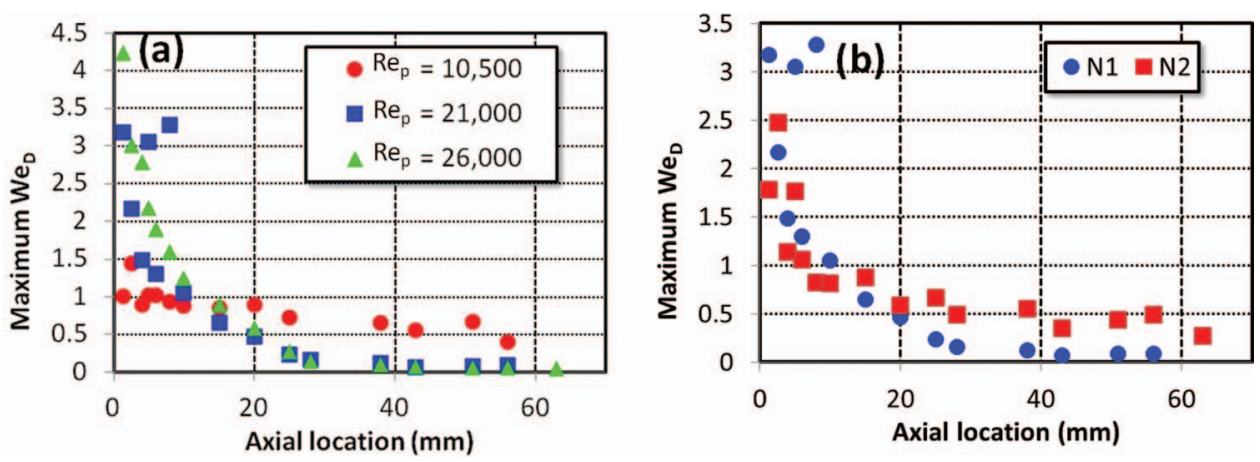

FIG. 17. Maximum $W e_{D}$ at different axial locations (a) for three Reynolds numbers (Nozzle N1), (b) for nozzles N1 and N2 at $\operatorname{Re}_{\mathrm{p}}=21000$. 
(a)

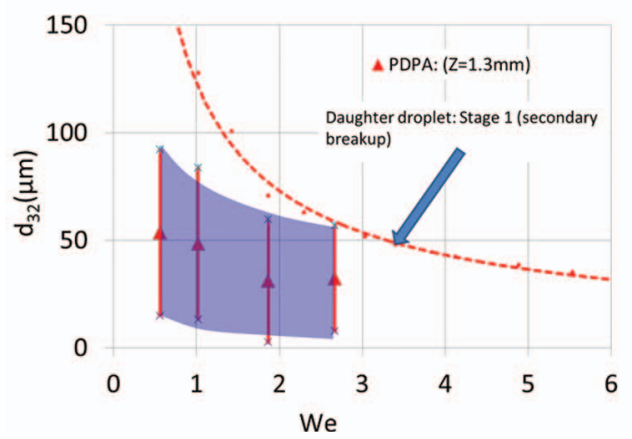

(b)

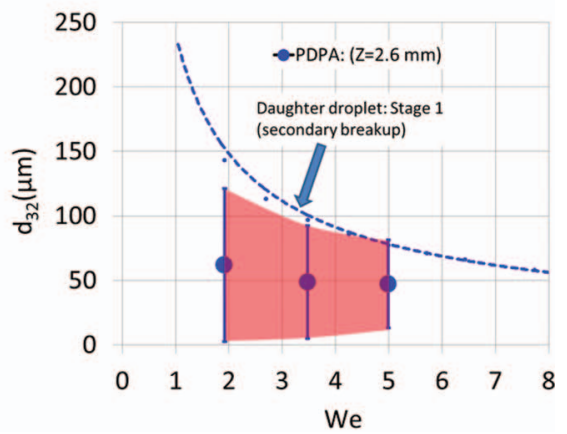

FIG. 18. Comparison of Sauter mean diameter (SMD) between theory (after secondary breakup: stage 1) and PDPA measurement at (a) $\mathrm{Z}=1.3 \mathrm{~mm}$ nozzle $\mathrm{N} 1$, (b) $\mathrm{Z}=2.6 \mathrm{~mm}$ nozzle $\mathrm{N} 2$. The PDPA data also show the $+/-3 \sigma$ range of the distribution.

the daughter droplets exhibit no such characteristics: ${ }^{31}$

$$
d_{32}=\frac{d_{D}}{1+\frac{8 K y^{2}}{20}+\frac{\rho_{l}\left(d_{D} / 2\right)^{3}(d c / d t)^{2}}{\sigma}\left(\frac{6 K-5}{120}\right)},
$$

where $K$ is a factor (of the order of 10/3) that describes the ratio between energy due to distortion and energy contained in the fundamental mode of the parent droplet, ${ }^{31} y$ is the non-dimensional deformation of the droplet radius, and $d_{32}$ is the Sauter mean diameter of the daughter droplet distribution. ${ }^{31}$ In the TAB model, the droplet deformation is measured by $\mathrm{x}$, which is the change in horizontal radius (undisturbed radius, $r$ ) of the droplet from its undisturbed radius. $y=x /\left(r . C_{r}\right.$ ), where $r . C_{r}$ represents the critical value of the $\mathrm{x}$, beyond with droplet deformation will result in breakup. Normally, $\mathrm{C}_{\mathrm{r}}=0.5$ under the assumption that maximum distortion allowed is equal to the droplet radius. A value of $\mathrm{y}=1$ signifies that the droplet deformation is equal to the critical threshold needed for the breakup.

Hsiang and Faeth ${ }^{32}$ and Guildenbecher et al. ${ }^{30}$ showed that for this range of $W e_{D}$ and $O h_{D}$, the aspect ratio and droplet deformation linearly changes with time. For an order of magnitude calculation we neglect the $3 \mathrm{rd}$ term in the denominator, which is expected to be smaller than other two terms due to the presence of $d_{D}{ }^{3}$. Here droplet diameter, $d_{D}$, is in the order of microns. Thus, an order of magnitude analysis shows that Eq. (8) can be written as $d_{32} \approx \frac{d_{D}}{1+\frac{8 K^{2}}{20}}$. With the assumptions that $\mathrm{K} \sim 10 / 3^{31}$ and $\mathrm{y}=1$ for breakup to occur we deduce $d_{32} \sim d_{D} / 2.33$. Figure 18 shows the calculated Sauter mean diameter of the daughter droplets after the first secondary breakup for both the nozzles. The plot also shows the Sauter mean diameter of first measurements after the film length ( $\mathrm{Z}=1.3$ and $2.6 \mathrm{~mm}$ for $\mathrm{N} 1$ and $\mathrm{N} 2$, respectively) for both the nozzles. The proposed simplified theoretical formulation of Sauter mean diameter is a conservative estimate and is used only for order of magnitude analysis.

Theoretically, the liquid film goes through different stages of breakup as the droplet moves downstream (shown in Figure 11). However, the first few stages occur almost simultaneously near the film length, making it difficult to identify a fixed Z-location where only primary or even first stage of secondary breakup occurs. The predicted diameters for first theoretical stage of secondary breakup are plotted in Figure 18. The first stage breakup profile yields higher theoretical diameters compared to the first measurement possible in the spray and decreases with Weber number, We (Weber number based on liquid film). The first stage breakup measurements are difficult to make using optical diagnostics due to the coexistence of the film and ligaments. The first set of PDPA measurements beyond the liquid film zone is made at $\mathrm{Z}=1.3 \mathrm{~mm}$ for the smaller nozzle (N1) and at $\mathrm{Z}=2.6 \mathrm{~mm}$ for the larger (N2) nozzle. The PDPA measurements at any location can be statistically described by a Sauter mean diameter along with a diameter distribution $(+/-3 \sigma$ of the measured droplet diameters). Hence, the expected diameter distribution after first stage of secondary 
breakup is well represented by the PDPA data as shown in Figure 18. These measured range of droplet diameters at $1.3 \mathrm{~mm}$ and $2.6 \mathrm{~mm}$ for $\mathrm{N} 1$ and $\mathrm{N} 2$ are smaller than the theoretical Sauter mean diameter estimated after the first stage of breakup. But the difference diminishes with increase in We. Thus, it is possible that the first two stages of secondary breakup occur before reaching these measurement points.

To summarize our finding on the secondary breakup, PDPA seems to be capable of making measurements of Stage 2 of breakup and compares with the theory. It is also seen that for lower Weber number (lower pressure), the two stages of secondary breakup are likely to occur before the droplets travel to reach the location of $1.3 \mathrm{~mm}$ for $\mathrm{N} 1$ and $2.6 \mathrm{~mm}$ for $\mathrm{N} 2$ from the nozzle tip. However, for higher pressures ( $>2 \mathrm{MPa}$ ) the theoretical first generation (stage 1) droplet diameter approaches the measured value, suggesting that there is likely to be only onset of secondary breakup prior to $\mathrm{Z}=1.3 \mathrm{~mm}$ for $\mathrm{N} 1$ and $\mathrm{Z}=2.6 \mathrm{~mm}$ for $\mathrm{N} 2$. This is expected as the droplet velocities increase with pressure, resulting in lower transition time from nozzle tip to reach these points of measurement.

\section{Coalescence dynamics}

When two droplets collide in a spray, either they bounce off each other or they coalesce. As the outcome largely depends on collision Weber number $\left(W e_{c o l}=\rho_{l} U_{r e l}^{2} d_{0} / \sigma_{l}\right.$, where $\rho_{l}$ is the liquid density; $d_{\mathrm{o}}$ is the droplet diameter; $U_{\text {rel }}$ is the relative velocity between the colliding droplets; and $\sigma_{l}$ is the liquid surface tension) and impact parameter (B). Jiang et al. ${ }^{33}$ and Qian and Law ${ }^{34}$ constructed regime diagram of collision outcome based on these two non-dimensional numbers. Qian and $\mathrm{Law}^{34}$ showed that coalescence occurrence depends on the rate of dissipation of collision kinetic energy through liquid viscosity after the two droplets collide. Thus, for head-on collision (B $=0$ ) of same size droplets coalescence will occur if the collision Weber number $\left(W e_{\text {col }}\right)$ is less than a critical value ( $W e_{\text {crit }}$ ), which depends on liquid viscosity and can be expressed as $W e_{\text {crit }}=30 . O h_{\text {col }}$ +15 , where $O h_{\text {col }}$ is the collision Ohnesorge number defined by $\left(O h_{c o l}=16 \mu_{l} /\left(\rho_{l} \sigma d_{0}\right)^{1 / 2}\right)$. In the current work, the probability of coalescence is calculated based on this principal. For a conservative estimate, we considered only head-on collision of the same size droplet, for which the relative velocity will be twice that of the individual droplet velocity. Using PDPA data for 10000 samples at each measurement location, we calculated $W e_{\text {col }}$ and $W e_{\text {crit }}$ for each individual droplets to evaluate statistical probability of $W e_{\text {col }}<W e_{\text {crit }}$, at which coalescence occurs. Mathematically, the coalescence probability can be cast as

$$
\text { Probability of Coalescence }=\frac{\text { Number of droplets with } W e_{c o l} \leq W e_{c r i t}}{\text { Total number of droplets }} .
$$

Note that in a spray, different droplets may possess all three velocity components, albeit with strong downward axial component. Thus, there can be four different types of collisions, namely, (a) same size, (b) different size head-on collisions $(\mathrm{B}=0)$, (c) same size, and (d) different size

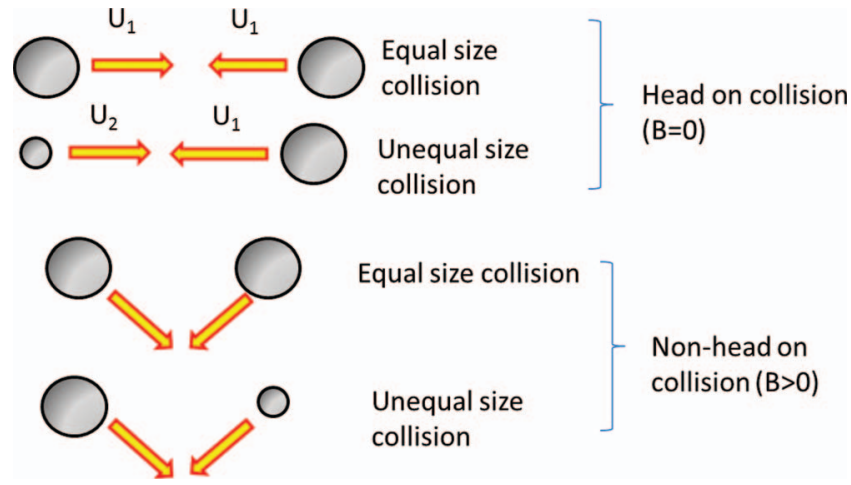

FIG. 19. Different types of droplet collisions. 

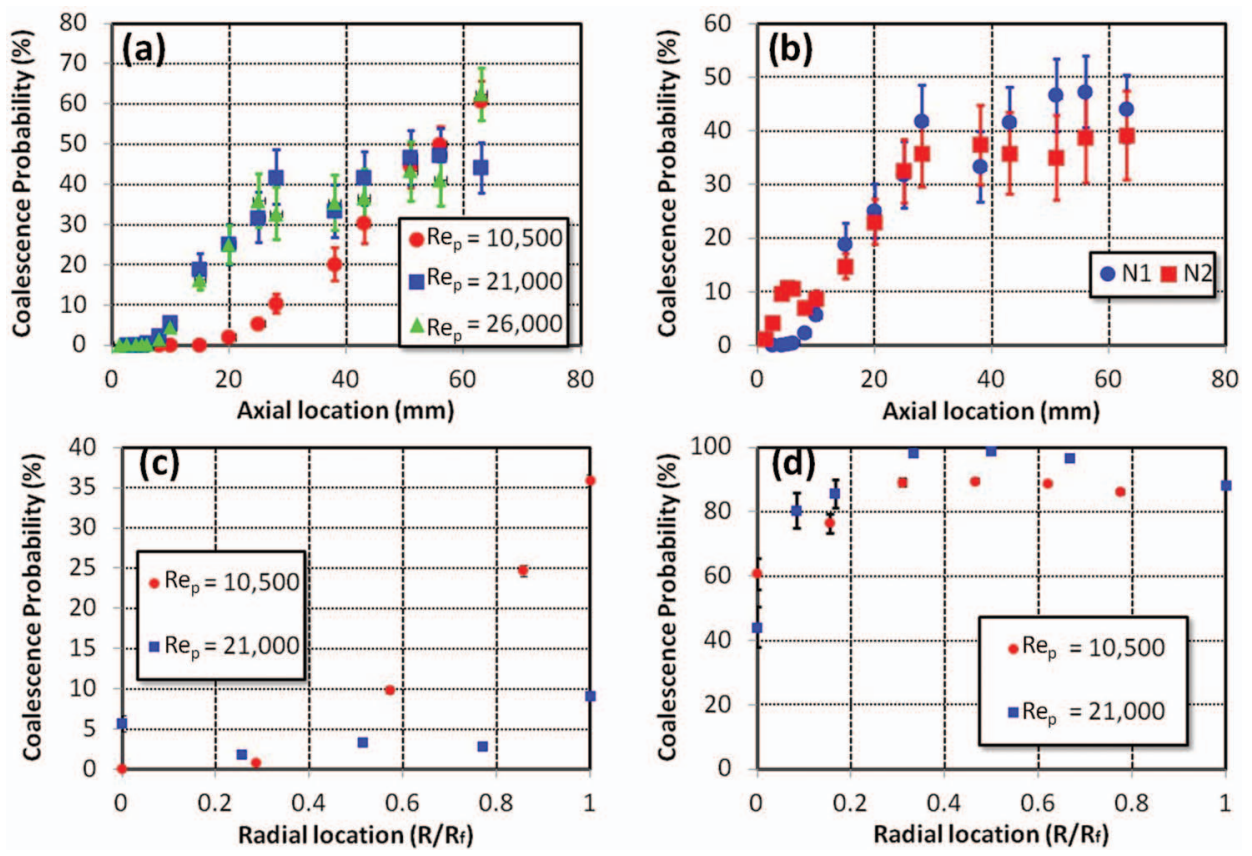

FIG. 20. The coalescence probability along the centerline of the spray at different axial locations (a) for nozzle N1 at different Reynolds numbers, (b) for nozzles N1 and N2 at $\mathrm{Re}_{\mathrm{p}}=21000$. Probability of coalescence at different radial locations of Nozzle N1 at two different Reynolds numbers (c) $Z=10 \mathrm{~mm}$ and (d) $Z=63 \mathrm{~mm}$.

off-center and non-head-on collisions $(\mathrm{B}>0)$, as shown in Figure 19. For the same size droplet collision of water, nevertheless, head-on collisions provide more chances of separation. For other types of collisions, the critical or transition Weber number increases with impact parameter (B) and large to small droplet size ratio increasing the probability of coalescence. In other words, the reported probability in this work is a first order estimate and a conservative calculation which represents a lower bound.

Based on this analysis, the collision probability is calculated and shown in Figure 20 for Nozzle $\mathrm{N} 1$ at different Re along the axial distance from the nozzle tip. The probability is very low close to the nozzle, reducing the chances of diameter increase through coalescence. At higher $\mathrm{Z}$ locations, i.e., $\mathrm{Z}>15 \mathrm{~mm}$, on the other hand, the probability of coalescence continues to increase with $\mathrm{Z}$ and plays a major role in increasing the droplet mean diameter as seen in Figure 20(a). The coalescence probability of the droplets along the radial location at $Z=10$ and $63 \mathrm{~mm}$ are shown in Figures 20(c) and 20(d). The probability increases significantly with radius due to the presence of smaller droplets with lower velocity. Furthermore, we also notice that different radial locations at $Z=63$ show higher coalescence probability compared to $\mathrm{Z}=10 \mathrm{~mm}$, due to lower axial velocity, thus, lower collision Weber number $\left(W e_{\text {col }}\right)$. Nozzle N2 shows very similar coalescence probability when compared to N1 nozzle (Figure 20(b)). In reality, the coalescence probability at the outer radius of the spray will be higher due to strong tangential component resulting in frequent off-center and unequal droplet collisions. The coalescence results are consistent with the observations in Figure 2 which show an initial decrease in diameter and then a steady increase around $Z=15-20 \mathrm{~mm}$. Figure 20 shows that the first order estimate of coalescence probability exhibits a significantly high magnitude around $\mathrm{Z}=20 \mathrm{~mm}$ for Reynolds numbers of 21000 and 26000 . The coalescence probability is quite low for $\mathrm{Z}<10 \mathrm{~mm}$ signifying a regime dominated by breakup and centrifugal dispersion. For $\operatorname{Re}_{\mathrm{p}}$ $=10500$, Figure 2 suggests that the diameter inflection occurs around $Z=35-40 \mathrm{~mm}$. Figure 20(a) corroborates the fact that this inflection is indeed a coalescence effect since the probability increases to $30 \%$ at $\mathrm{Z} \sim 40 \mathrm{~mm}$ for $\mathrm{Re}=10500$. Similar effect can be seen for both the nozzles N1 and N2 (Figure 20(b)). 
The variation of coalescence probability in the radial direction is reported in Figs. 20(c) and 20(d) for nozzle N1. Coalescence probability shows steady increase in the radial direction particularly at $\mathrm{Z}=63 \mathrm{~mm}$ (Figure 20(d)). At $\mathrm{Z}=63 \mathrm{~mm}$, it is expected that both primary and secondary breakup mechanisms are very weak as evident in Figs. 2(a), 8(c), and 10(c). However, the low velocity scale $(\sim 2 \mathrm{~m} / \mathrm{s})$ aids in vigorous coalescence as seen in Figure 20(d). Hence, coalescence will be very strong at all radial locations at $\mathrm{Z}=63 \mathrm{~mm}$. This is further corroborated by the steady increase in average droplet diameter in the radial direction as seen in Figures 8(c) and 10(c). At $\mathrm{Z}=10 \mathrm{~mm}$, the coalescence probability is much lower at all radial locations since the velocity scale is still very high ( $\sim 25 \mathrm{~m} / \mathrm{s})$ as seen in Figs. 6(b) and 9(b). The resultant diameters (Figures 6(c) and $9(\mathrm{c}))$ are also almost two times lower at $\mathrm{Z}=10 \mathrm{~mm}$ at all radial locations compared to those for $Z=63 \mathrm{~mm}$. This further indicates lower coalescence probability which is shown in Figure 20(c). At $\mathrm{Z}=10 \mathrm{~mm}$, we see that the coalescence probability is lower for higher Reynolds number (Figure 20(c)). Higher Reynolds number induces stronger swirl which is due to an increase in the spray cone angle. Pressure swirl pushes the larger droplets towards the outer periphery of the spray. Thus, at higher Reynolds number and stronger swirl intensity, number of larger droplets increases towards the outer periphery of the spray, resulting in low coalescence probability.

\section{Centrifugal effects due to pressure swirl}

The other aspect which needs to be considered is the effect of pressure swirl on the droplet distribution. In a pressure-swirl atomizer the internal design of the nozzle imposes a tangential motion on the liquid film, which imparts a tangential velocity component on the droplets. Although the axial motion is stronger than its tangential counterpart, this pressure swirl affects the droplet diameter distribution in the spray cone. The swirling motion of the droplets creates a centrifugal effect and moves the larger droplets towards the perimeter of the spray. If we consider a balance between the centrifugal force $\left(F_{c}\right)$ acting radially outward and the aerodynamic drag force $\left(F_{C D}\right)$ acting against that motion, $F_{C}=\frac{\rho_{l} \pi d_{0}^{3} V_{T}^{2}}{6 R}$ and $F_{C D}=\frac{C_{D T} \rho_{g} \pi d_{o}^{2} V_{R}^{2}}{8}$, where $V_{T}$ and $V_{R}$ are the tangential and radial component of the velocity due to pressure swirl; $C_{D T}$ is the drag coefficient against radial motion; $\rho_{g}$ and $\rho_{l}$ are gas and liquid density; $d_{\mathrm{o}}$ is the droplet diameter; and $R$ is the radial location. Now, computing Reynolds number based on radial velocity, $\operatorname{Re}_{R D}=\frac{\rho_{g} d_{0} V_{R}}{\mu_{g}}<1, C_{\mathrm{DT}}=24 / \mathrm{Re}_{\mathrm{RD}}$, and $F_{C D}=3 \mu_{g} \pi d_{0} V_{R}$. At equilibrium, $F_{C}=F_{C D}$ and $V_{R}=\frac{\rho_{l} V_{T}^{2}}{18 \mu_{l}} \cdot \frac{d_{0}^{2}}{R}$. This shows that the radial velocity at any given radius is stronger for a larger droplet. When the droplets move downwards, larger droplets move towards outer periphery of the spray depleting the larger droplets at the centerline. Thus, the average droplet diameter along the centerline sharply drops. This is corroborated by the decrease in droplet diameter in Figure 2 beyond $Z=5-10 \mathrm{~mm}$, even though primary and secondary breakup mechanisms are much weaker and coalescence probability is significantly low for all Reynolds numbers.

If we combine the effects of coalescence, breakup, and centrifugal dispersion, we can divide the spray in three zones as shown in Figure 3. The first zone, or zone A, is very close to the nozzle and consists predominantly of film and ligament regime, where primary breakup is very dominant with some secondary breakup as shown by $W e_{D} \sim 1$ and small breakup time, $\mathrm{t}_{\mathrm{b}}$ (Figure 16). In zone $\mathrm{B}$, the secondary breakup process continues. But the process becomes weaker as shown by larger breakup time, $\mathrm{t}_{\mathrm{b}}$. The centrifugal dispersion, however, becomes dominant removing the larger droplets towards the outer periphery of the spray and we see sharp decrease in droplet diameter. For both the zones, A and B, the coalescence process is very weak due to high collision Weber number $\left(W e_{\text {col }}\right.$ ) reflected by lower coalescence probability (Figure 20 ). However, beyond $\mathrm{Z} \sim 15 \mathrm{~mm}$, this process becomes stronger and dominant over other competing effects of breakup and centrifugal dispersion. Thus, we observe droplet diameter to increase sharply beyond this point. This zone with high probability of coalescence is labeled as Zone C.

\section{CONCLUSION}

The breakup characteristics of two hydraulic injector nozzles were studied experimentally as a function of Reynolds number and Weber number both axial and radially. These two nozzles 
were classified as $\mathrm{N} 1$ and $\mathrm{N} 2$ with orifice diameter $0.3 \mathrm{~mm}$ and $0.5 \mathrm{~mm}$, respectively. Liquid was injected into the testing zone at injection pressures ranging from 0.3 to $4 \mathrm{MPa}$ (Reynolds number range of 7000-26000). This experimental study consisted of using two laser diagnostic techniques, shadowgraph and PDPA to understand the breakup characteristic effects as a function of Reynolds number and Weber number. The findings may be summarized as follows.

The two nozzles experienced two different types of wavelength profiles which are dependent on Reynolds number. Two types of wave breakup were observed and matched well with theory. Both values reached an asymptotic value in direct relationship with the spray cone angle which also reached a constant value beyond a certain Reynolds number. It was observed that the velocity and average diameter profiles are highly dependent on the non-dimensional mass flow rate or discharge coefficient and Reynolds number. In the axial direction, the average diameter decreased clearly illustrating different breakup regimes. Reynolds number shifts the transition from one regime to another closer to the nozzle tip with a diameter decrement along the centerline. The larger nozzle (N2) generated larger diameters. The velocity had a constant decreasing slope for the first $25.4 \mathrm{~mm}$ of the spray trajectory at which point the slopes decreased and became uniform towards the end. For lower Reynolds numbers and lower discharge coefficient in the radial direction, the data rate, velocity, and average diameter decreased linearly due to the film lengths intersecting at axial location $10 \mathrm{~mm}$. This behavior caused a shift in diameter profile due to increased probability of coalescence. Likewise, for higher Reynolds numbers and lower discharge coefficients, the spray resembled a more traditional hollow cone where the peak data rate occurs in the middle of the spray. At these conditions, it was also shown that the velocity transformed from a parabolic profile to a logarithmic decay at different axial locations due to coalescence. This was also reflected in the average drop diameter which increased for most cases.

The comparison of critical wavelength from high speed images with the theoretical analysis shows good comparison. The long and short wavelength breakup show that the ligaments breakup into droplets of size $\sim 500 \mu \mathrm{m}$. These droplets further disintegrate through a vibrational type of breakup and create smaller droplet. The PDPA measurement was not possible at this location. Comparison of daughter droplet diameter with the first measurement shows that for lower Weber numbers, there are successive secondary breakups for both the nozzles before axial location of $1.3 \mathrm{~mm}$. Stage 2 breakup compared well with the theory for both nozzles at this first location. At higher Weber numbers (higher pressure), droplets undergo only a single breakup prior to this measurement location. The actual estimation of the droplet size distribution requires a full scale simulation of the spray modeling which could be validated using current measurements. In this work, a simple analysis shows that the droplet size distribution for different pressure or Weber number follows the breakup models closely.

The paper also subdivides the spray into three regimes: near the nozzle, a zone consisting of film and ligament regime, where primary breakup and some secondary breakup take place, where $W e_{D} \sim 1$ and breakup time, $\mathrm{t}_{\mathrm{b}}$, is small; a second zone where the secondary breakup process continues, but weakens as the breakup time becomes larger, and the centrifugal dispersion becomes dominant removing the larger droplets to the spray perimeter; and finally, a zone away from the spray $(>15 \mathrm{~mm})$ where coalescence is dominant and the droplet diameters begin to increase.

\section{ACKNOWLEDGMENTS}

We would like to thank Parker Hannifin for providing UCF with their injector nozzles for the experiments, funded by AFRL. Funding from CSIR, India is acknowledged.

\footnotetext{
${ }^{1}$ H. Shi and C. Kleinstreuer, "Simulation and analysis of high-speed droplet spray dynamics," J. Fluids Eng. 129, 621-633 (2007).

${ }^{2}$ A. H. Lefebvre, Atomization and Sprays (Combustion: An International Series) (CRC, Boca Raton, FL, 1989).

${ }^{3}$ P. K. Senecal, D. P. Schmidt, I. Nouar, C. J. Rutland, and R. D. Reitz, "Modelling high-speed viscous liquid sheet atomization," Int. J. Multiphase Flow 25, 1073-1097 (1999).

${ }^{4}$ D. P. Schmidt, I. Nouar, P. K. Senecal, J. Homan, C. J. Rutland, J. Martin, and R. D. Reitz, "Pressure-swirl atomization in the near field," SAE Paper 1999-01-0496 (1999).

${ }^{5}$ A. Mansour and N. Chigier, "Dynamic behavior of liquid sheets," Phys. Fluids 3, 2971-2980 (1991).

${ }^{6}$ G. M. Faeth, L. P. Hsiang, and P. K. Wu, "Structure and breakup properties of sprays," Int. J. Multiphase Flow 21, 99-127 (1995).
} 
${ }^{7}$ S. H. Park, H. J. Kim, H. K. Suh, and C. S. Lee, "Experimental and numerical analysis of spray-atomization characteristics of biodiesel fuel in various fuel and ambient temperatures conditions," Int. J. Heat Fluid Flow 30, 960-970 (2009).

${ }^{8}$ H. J. Kim, H. K. Suh, S. H. Park, and C. S. Lee, "An experimental and numerical investigation of atomization characteristics of biodiesel, dimethyl ether, and biodiesel-ethanol blended fuel," Energy Fuels 22, 2091-2098 (2008).

9 R. Payri, B. Tormos, F. J. Salvado, and L. Araneo, "Spray droplet velocity characterization for convergent nozzles with three different diameters," Fuel 87, 3176-3182 (2008).

${ }^{10}$ A. Tratnig, G. Brenn, T. Stirxner, P. Franhauser, N. Laubacher, and M. Stranzinger, "Characterization of spray formation from emulsions by pressure-swirl atomizers for spray drying," J. Food Eng. 95, 126-134 (2009).

${ }^{11}$ A. Aliseda, E. J. Hopfinger, J. C. Lasheras, D. M. Kremer, A. Berchielli, and E. K. Connolly, "Atomization of viscous and non-newtonian liquids by a coaxial, high-speed gas jet. Experiments and droplet size modelling," Int. J. Multiphase Flow 34, 161-175 (2008).

${ }^{12}$ M. C. Butler Ellis, C. R. Tuck, and P. C. H. Miller, "Dilute emulsions and their effect on the breakup of the liquid sheet produced by flat-fan spray nozzles," Atomization Sprays 9, 385-397 (1999).

${ }^{13}$ C. D. Bolszo, M. Rohani, A. A. Narvaez, D. Dunn-Rankin, V. G. McDonell, and W. A. Sirignano, "Pressure-swirl atomization of water-in-oil emulsions," Atomization Sprays 20, 1077-1099 (2010).

${ }^{14}$ M. Ahmed, N. Ashgriz, and H. N. Tran, "Break-up length and spreading angle of liquid sheets formed by splash plate nozzles,” J. Fluids Eng. 131, 011306-1-011306-9 (2009).

${ }^{15}$ R. Reitz and F. V. Bracco, "Mechanism of atomization of a liquid jet," Phys. Fluids 25, 1730-1742 (1982).

${ }^{16}$ S. P. Lin and R. D. Reitz, "Drop and spray formation from a liquid jet," Annu. Rev. Fluid Mech. 30, 85-105 (1998).

${ }^{17}$ W. A. Sirignano and C. Mehring, "Review of theory of distortion and disintegration of liquid streams," Prog. Energy Combust. Sci. 26, 609-655 (2000).

${ }^{18}$ J. C. Lasheras, E. Villermaux, and E. J. Hopfinger, "Break-up and atomization of a round water jet by a high-speed annular air jet," J. Fluid Mech. 357, 351-379 (1998).

${ }^{19}$ J. C. Lasheras and E. J. Hopfinger, "Liquid jet instability and atomization in a coaxial gas stream," Annu. Rev. Fluid Mech. 32, 275-308 (2000).

${ }^{20} \mathrm{C}$. Dumouchel, "Measurements of breakup length of cylindrical liquid jets. Application to low-pressure car injector," Atomization Sprays 11, 201-226 (2001).

${ }^{21}$ C. Dumouchel, "On the experimental investigation on primary atomization of liquid streams," Exp. Fluids 45, 371-422 (2008).

${ }^{22}$ C. Dumouchel, J. Cousin, and K. Triballier, "On the role of the liquid flow characteristics on low-Weber-number atomization processes," Exp. Fluids 38, 637-647 (2005).

${ }^{23}$ D. Sivakumar and V. Kulkarni, "Regimes of spray formation in gas-centered swirl coaxial atomizers," Exp. Fluids 51, 587-596 (2011).

${ }^{24}$ S. Wahono, D. Honnery, J. Soria, and J. Ghojel, "High speed visualisation of primary break-up of an annular liquid sheet," Exp. Fluids 44, 451-459 (2008).

${ }^{25}$ P. Walzel, "Spraying and atomizing of liquids," Ullmann's Encyclopedia of Industrial Chemistry (Wiley, 2010)

${ }^{26}$ A. Lichtarowicz, R. K. Duggins, and E. Markland, "Discharge coefficients for incompressible non-cavitating flow through long orifices," J. Mech. Eng. Sci. 7, 210-219 (1965).

${ }^{27}$ L. Rayleigh, "On the instability of jets," Proc. London Math. Soc. 10, 4-13 (1879).

${ }^{28}$ N. Dombrowski and W. R. Johns, "The aerodynamic instability and disintegration of viscous liquid sheets," Chem. Eng. Sci. 18, 203-214 (1963).

${ }^{29}$ C. Weber, "On the breakdown of a liquid jet," Z. Angew. Math. Phys. 11, 136-159 (1931).

${ }^{30}$ D. R. Guildenbecher, C. Lopez-Rivera, and P. E. Sojka, "Secondary atomization," Exp. Fluids 46, 371-402 (2009).

${ }^{31}$ P. J. O' Rourke and A. A. Amsden, "The TAB method for numerical calculation of spray droplet breakup," SAE Technical Paper 872089 (1987).

${ }^{32}$ L. P. Hsiang and G. M. Faeth, "Near-limit drop deformation and secondary breakup," Int J. Multiphase Flow 18, 635-652 (1992).

${ }^{33}$ Y. J. Jiang, A. Umemura, and C. K. Law, "An experimental investigation on the collision behavior of hydrocarbon droplets," J. Fluid Mech. 234, 171-190 (1992).

${ }^{34}$ J. Qian and C. K. Law, "Regimes of coalescence and separation in droplet collision,” J. Fluid Mech. 331, 59-80 (1997). 